\title{
Ramond-Ramond gauge fields in superspace with manifest T-duality
}

\author{
Machiko Hatsuda, ${ }^{a, b}$ Kiyoshi Kamimura $^{c}$ and Warren Siegel ${ }^{d, 1}$ \\ a Physics Division, Faculty of Medicine, Juntendo University, \\ Chiba 270-1695, Japan \\ ${ }^{b}$ KEK Theory Center, High Energy Accelerator Research Organization, \\ Tsukuba, Ibaraki 305-0801, Japan \\ ${ }^{c}$ Department of Physics, Toho University, \\ Funabashi 274-8510, Japan \\ ${ }^{d}$ C.N. Yang Institute for Theoretical Physics, State University of New York, \\ Stony Brook, NY 11794-3840, U.S.A. \\ E-mail: mhatsuda@post.kek.jp, kamimura@ph.sci.toho-u.ac.jp, \\ siegel@insti.physics.sunysb.edu
}

ABSTRACT: A superspace with manifest T-duality including Ramond-Ramond gauge fields is presented. The superspace is defined by the double nondegenerate super-Poincaré algebras where Ramond-Ramond charges are introduced by central extension. This formalism allows a simple treatment that all the supergravity multiplets are in a vielbein superfield and all torsions with dimension 1 and less are trivial. A Green-Schwarz superstring action is also presented where the Wess-Zumino term is given in a bilinear form of local currents. Equations of motion are separated into left and right modes in a suitable gauge.

KeYwords: Superspaces, Supersymmetry and Duality, Space-Time Symmetries, String Duality

ARXIV EPRINT: 1411.2206

\footnotetext{
${ }^{1}$ http://insti.physics.sunysb.edu/ siegel/plan.html.
} 


\section{Contents}

1 Introduction 1

2 Algebras 4

2.1 Central extension of type II super-Poincaré algebra 4

2.2 Central extension of affine type II super-Poincaré algebra 5

$3 \quad \kappa$-symmetric Virasoro constraints $\quad 6$

$3.1 \kappa$-symmetry 7

$3.2 \kappa$-symmetric Virasoro constraints 8

4 Torsion and vielbein $\quad 9$

4.1 Torsion constraints 9

$\begin{array}{lll}4.2 & \text { Vielbein } & 11\end{array}$

$\begin{array}{lll}4.3 & \text { Linearized solution } & 14\end{array}$

5 Green-Schwarz superstring action $\quad 16$

$\begin{array}{lll}5.1 \text { Action } & 16\end{array}$

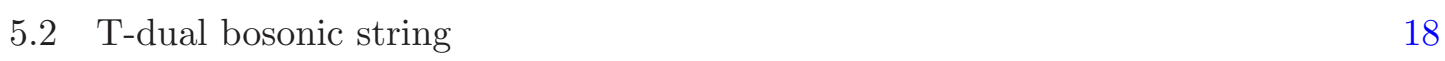

5.3 Type II superstring 20

5.4 Type II superstring with R-R extension 22

6 Conclusions and discussions $\quad 23$

\section{Introduction}

T-duality is one of the most important key ingredients of the gravity theory beyond general relativity which may be the superstring theory. The T-duality was realized manifestly by doubling coordinates firstly in [1-3]. T-duality covariant Lie bracket proposed in [1-3] is turned out to be reduced into the Courant bracket introduced in $[4,5]$ to describe generalized geometry. Supersymmetric extension of T-duality symmetry with RamondRamond (R-R) fields has been studied in [6-10]. This subject is broaden in [11-14] and its recent development is in review articles for example [15-17].

A superspace approach of type II supergravity theories with manifest T-duality has been proposed in $[18,19]$. Supergravity theories were constructed in superspace with almost all symmetries manifest except T-duality symmetry in [20-22]. Our superspace involves two kinds of doubling in order to realize manifest T-duality: (1) Nondegenerate algebra by introducing nondegenerate pairs of generators such as $\left(D_{\mu}, \Omega^{\mu}\right)[23-26]$ and $\left(S_{m n}, \Sigma^{m n}\right)$ [27-31], (2) Double coordinate space by using momenta and winding modes such as $\left(P_{m}, P_{m^{\prime}}\right)$ [1-3]. The doubling $(1)$ is required from consistency of the 
stringy general coordinate invariance. The Lorentz generator and its nondegenerate partner $\left(S_{m n}, \Sigma^{m n}\right)$ are also basis in order to make Lorentz connection to be a part of the vielbein fields [31]. It may be useful for writing T-duality on the worldsheet. It is also possible to write the WZ term as bilinears of currents for arbitrary backgrounds. This doubling is resolved by dimensional reduction constraints. Double coordinate space (2) realizes a simpler and unified treatment of torsion constraints and vielbein. This doubling is resolved by imposing the section condition and the strong constraint.

In this paper we include the R-R gauge fields to complete the construction of the superspace of the type II supergravity. The R-R gauge fields are necessary as a type II superstring background, although a superstring does not carry R-R charges. The R-R gauge fields couple to $\mathrm{D} p$-branes, where $p$ is even for type IIA and odd for type IIB theories. Brane charges are included by central extension of superalgebras [32-35]. Usually these brane algebras are realized on worldvolumes. Their generalized Lie bracket and gauge transformations of brane gauge fields are obtained in [36-38]. In this paper the R-R charges, denoted by $\Upsilon_{\mu \nu^{\prime}}$, are introduced by central extension of a superstring algebra. Or equivalently $\Upsilon_{\mu \nu^{\prime}}$ is introduced in an affine algebra for the double coordinate space by central extension. This central charge $\Upsilon_{\mu \nu^{\prime}}$ is constrained to be zero on the superstring worldsheet, but $\Upsilon_{\mu \nu^{\prime}}$ is necessary to describe the R-R gauge fields and its gauge symmetries.

Inclusion of the R-R fields distinguishes T-duality between IIA and IIB superstring theories in our formalism. Under a discrete T-duality transformation IIA theory and IIB theory are interchanged and a $\mathrm{D} p$-brane changes to a $\mathrm{D}(p \pm 1)$-brane. This is explained from the type II superstring algebra in double space as follows: there are two irreducible supertranslation algebras in a type II superstring theory

$$
\begin{aligned}
\left\{D_{\mu}, D_{\nu}\right\} & =2 P_{m} \gamma^{m}{ }_{\mu \nu}, & \left\{D_{\mu^{\prime}}, D_{\nu^{\prime}}\right\} & =2 P_{m^{\prime}} \gamma^{m^{\prime} \nu_{\nu^{\prime}}} \\
P_{m} & =p_{\mathrm{m}}+\partial_{\sigma} x^{\mathrm{m}}, & P_{m^{\prime}} & =p_{\mathrm{m}}-\partial_{\sigma} x^{\mathrm{m}} .
\end{aligned}
$$

Usual Lorentz vector index is denoted by $p_{\mathrm{m}}$. Since in our approach two independent Lorentz generators are used, $S_{m n}$ for $P_{m}$ and $D_{\mu}$ and $S_{m^{\prime} n^{\prime}}$ for $P_{m^{\prime}}$ and $D_{\mu^{\prime}}$, two chiralities of two chiral spinors $D_{\mu}$ and $D_{\mu^{\prime}}$ are not fixed. The type of theories, IIA or IIB, is determined after reducing two Lorentz symmetries into one Lorentz symmetry. A discrete T-duality transformation in $\mathbf{n}$-th direction, which is an interchange between $p_{\mathbf{n}}$ and $\partial_{\sigma} x^{\mathbf{n}}$, is expressed in terms of an unit vector $n^{m}=\delta^{m \mathbf{n}}$ and $n^{m^{\prime}}=\delta^{m^{\prime} \mathbf{n}}$ as

$$
P_{m} \rightarrow P_{m}, P_{m^{\prime}} \rightarrow P_{m^{\prime}}-2 n_{m^{\prime}}\left(n^{l^{\prime}} P_{l^{\prime}}\right)
$$

It requires a change of chirality of $D_{\mu^{\prime}}$ as

$$
D_{\mu} \rightarrow D_{\mu}, D_{\mu^{\prime}} \rightarrow i D_{\nu} \eta^{\nu^{\prime} \mu^{\prime}}, \not \psi=n_{n^{\prime}} \gamma^{n^{\prime}} .
$$

The chirality of $(D \eta)^{\mu^{\prime}}$ is opposite to the one of $D_{\mu^{\prime}}$, since $\not$ anticommute with $\Gamma^{11}$. This is a parity transformation which is part of $\mathrm{O}(\mathrm{d}-1,1) \times \mathrm{O}(\mathrm{d}-1,1)$. The $\mathrm{R}-\mathrm{R}$ charge $\Upsilon_{\mu \nu^{\prime}}$ appears in a superalgebra between $D_{\mu}$ and $D_{\mu^{\prime}}$. The R-R charge $\Upsilon_{\mu \nu^{\prime}}$ is transformed under the discrete T-duality as

$$
\left\{D_{\mu}, D_{\nu^{\prime}}\right\}=2 \Upsilon_{\mu \nu^{\prime}} \rightarrow\left\{D_{\mu},(D \eta)^{\nu^{\prime}}\right\}=2(\Upsilon \not)_{\mu}{ }^{\nu^{\prime}}
$$


The R-R charges $\left(\Upsilon_{\eta}\right)_{\mu} \nu^{\nu^{\prime}}$ have different form numbers from $\Upsilon_{\mu \nu^{\prime}}$, when they are expanded by antisymmetric gamma matrix basis after the dimensional reduction. The form number is equal to $p$ for $\mathrm{D} p$-brane.

In this paper we extend the manifestly T-duality formalism of type II superspace [18] to the one with the R-R gauge fields. It requires the following new procedures;

- Central extension of the affine Lie algebra

- Torsion constraints from $\kappa$-symmetric Virasoro constraints and Bianchi identities involving R-R charges

- Field identification and solving torsion constraints including R-R fields

in addition to the previous procedures;

1. Construct an affine Lie algebra and double the generators.

2. Make covariant derivatives with the vielbein field $E_{\underline{A}} \underline{\underline{M}}$.

3. Find torsion constraints from $\kappa$-symmetric Virasoro constraints and Bianchi identities.

4. Solve torsion constraints with constraints for dimensional reduction and the isotropy group in addition to the section conditions (the strong constraint).

(a) Section conditions (the strong constraint): the section constraints (6.2) reduce doubled coordinates to half. These constraints give rise to the stringy contribution in the "new Lie derivative" [1-3, 18].

(b) Isotropy constraints: the isotropy group constraints are usual gauge constarints for a gauged coset model; such as Lorentz group parts $S_{m n}=S_{m^{\prime} n^{\prime}}=0$ for Poincaré groups [18, 31].

(c) Dimensional reduction constraints: dimensional reduction constraints are imposed on the unphysical symmetry currents to remove auxiliary coordinates which are introduced to make nondegenerate group metric; such as $\tilde{\Omega}^{\mu}=\tilde{\Omega}^{\mu^{\prime}}=$ $\tilde{\Sigma}^{m n}=\tilde{\Sigma}^{m^{\prime} n^{\prime}}=0[18,26,31,48]$.

The organization of the paper is the following: in next section the R-R charges are introduced by a central extension of the affine Lie algebra. In section $3 \kappa$-symmetric Virasoro constraints involving the R-R charges are obtained. In section 4 torsion constraints are determined from the $\kappa$-symmetric Virasoro constraints and Bianchi identities. Components of the supervielbein field are identified with the supergravity contents including the $\mathrm{R}-\mathrm{R}$ gauge fields. Torsion constraints are solved. In section 5 a Green-Schwarz superstring action in a manifestly T-duality formalism is given. The Wess-Zumino term is given in a bilinear form of currents and equations of motion are chirally separated in a suitable gauge. 


\section{Algebras}

In this paper we construct a superspace for the type II superstring background. The superspace is defined by affine nondegenerate double super-Poincaré algebras with the R-R central charges. Notation follows our previous paper [18]. After a central extension of a Lie algebra, its affine extension is presented.

\subsection{Central extension of type II super-Poincaré algebra}

We begin by two independent sets of super-Poincaré algebras generated by $p_{m}, s_{m n}$ with $m=0,1, \cdots, 9$ and $d_{\mu}$ with $\mu=1, \cdots, 16$ for left and $p_{m^{\prime}}, s_{m^{\prime} n^{\prime}}$ with $m^{\prime}=0,1, \cdots, 9$ and $d_{\mu^{\prime}}$ with $\mu^{\prime}=1, \cdots, 16$ for right. A central extension of the double super-Poincaré algebras is performed by introducing R-R charges denoted by " $\Upsilon_{\mu \nu}$ " in an anticommutator between $d_{\mu}$ and $d_{\mu^{\prime}}$. " $\Upsilon_{\mu \nu}$ " carries mixed left and right indices.

Generators of two sets of super-Poincaré algebras including $\Upsilon_{\mu \nu^{\prime}}$ are denoted by $G_{I}$. Its affine extension requires nondegenerate metric. The metric between $p_{m}$ 's and $p_{m^{\prime}}$ 's are usual metrics $\eta_{m n}$ and $\eta_{m^{\prime} n^{\prime}}$. In order to define a nondegenerate metric for $G_{I}$ except $p_{m}$ and $p_{m^{\prime}}$ we introduce $G_{\tilde{I}}$ :

\begin{tabular}{|c|c|c|c|c|}
\hline $\begin{array}{l}\text { dim. } \\
G_{I}\end{array}$ & $\begin{array}{c}0 \\
s_{m n}, s_{m^{\prime} n^{\prime}}\end{array}$ & $\begin{array}{c}\frac{1}{2} \\
d_{\mu}, d_{\mu^{\prime}}\end{array}$ & $\begin{array}{c}1 \\
\Upsilon_{\mu \nu^{\prime}}\end{array}$ & $\begin{array}{c}1 \\
p_{m}, p_{m^{\prime}}\end{array}$ \\
\hline dim. & 2 & $\frac{3}{2}$ & 1 & \\
\hline$G_{\tilde{I}}$ & $\sigma^{m n}, \sigma^{m^{\prime} n}$ & $\omega^{\mu}, \omega^{\mu^{\prime}}$ & $F^{\mu \nu^{\prime}}$ & \\
\hline
\end{tabular}

The sum of dimensions of a nondegenerate pair of generators is 2. Dimensions of $G_{I}$ are not greater than 1, while dimensions of $G_{\tilde{I}}$ are not less than 1 . The nondegenerate partner of the R-R central charge, $\Upsilon_{\mu \nu^{\prime}}$, is denoted by "F ${ }^{\mu \nu^{\prime}}$ (digamma)".

A double super-Poincaré algebra with central extension is generated by $G_{I}$

$$
\begin{array}{cl}
\operatorname{dim} 0:\left[s_{m n}, s_{l k}\right]=-i \eta_{[k \mid[m} s_{n] \mid l]} & {\left[s_{m^{\prime} n^{\prime}}, s_{l^{\prime} k^{\prime}}\right]=i \eta_{\left[k^{\prime} \mid\left[m^{\prime}\right.\right.} s_{\left.\left.n^{\prime}\right] \mid l^{\prime}\right]}} \\
\operatorname{dim} \frac{1}{2}:\left[s_{m n}, d_{\mu}\right]=-\frac{i}{2}\left(d \gamma_{m n}\right)_{\mu} & {\left[s_{m^{\prime} n^{\prime}}, d_{\mu^{\prime}}\right]=\frac{i}{2}\left(d \gamma_{m^{\prime} n^{\prime}}\right)_{\mu^{\prime}}} \\
\operatorname{dim} 1:\left[s_{m n}, p_{l}\right]=-i p_{[m} \eta_{n] l} & {\left[s_{m^{\prime} n^{\prime}}, p_{l^{\prime}}\right]=i p_{\left[m^{\prime}\right.} \eta_{\left.n^{\prime}\right] l^{\prime}}} \\
{\left[s_{m n}, \Upsilon_{\mu \nu^{\prime}}\right]=-\frac{i}{2} \Upsilon_{\rho \nu^{\prime}}\left(\gamma_{m n}\right)_{\mu}{ }_{\mu}\left[s_{m^{\prime} n^{\prime}}, \Upsilon_{\mu \nu^{\prime}}\right]=\frac{i}{2} \Upsilon_{\mu \rho^{\prime}}\left(\gamma_{m^{\prime} n^{\prime}}\right)_{\nu^{\prime}}^{\rho^{\prime}}} \\
\left\{d_{\mu}, d_{\nu}\right\}=2 p_{m} \gamma^{m}{ }_{\mu \nu} \quad\left\{d_{\mu^{\prime}}, d_{\nu^{\prime}}\right\}=-2 p_{m^{\prime}} \gamma_{\mu^{\prime} \nu^{\prime}} \\
\left\{d_{\mu}, d_{\nu^{\prime}}\right\}=2 \Upsilon_{\mu \nu^{\prime}}
\end{array}
$$

The gamma matrices satisfy

$$
\left(\gamma^{m}\right)_{\mu \nu}=\left(\gamma^{m}\right)_{\nu \mu}, \quad\left(\gamma^{(m \mid}\right)^{\mu \rho}\left(\gamma^{\mid n)}\right)_{\rho \nu}=2 \eta^{m n} \delta_{\nu}^{\mu}, \quad\left(\gamma_{m}\right)_{(\mu \nu}\left(\gamma^{m}\right)_{\rho) \lambda}=0
$$


The rest of the commutators including generators of $G_{\tilde{I}}$ is given by

$$
\begin{aligned}
& \operatorname{dim} 1:\left[s_{m n}, \mathrm{~F}^{\mu \nu^{\prime}}\right]=\frac{i}{2}\left(\gamma_{m n}\right)^{\mu}{ }_{\nu} \mathrm{F}^{\nu \nu^{\prime}}\left[s_{m^{\prime} n^{\prime}}, \mathrm{F}^{\mu \nu^{\prime}}\right]=-\frac{i}{2}\left(\gamma_{m^{\prime} n^{\prime}}\right)^{\nu^{\prime}{ }_{\mu^{\prime}} \mathrm{F}^{\mu \mu^{\prime}}} \\
& \operatorname{dim} \frac{3}{2}:\left[s_{m n}, \omega^{\mu}\right]=\frac{i}{2}\left(\gamma_{m n} \omega\right)^{\mu} \quad\left[s_{m^{\prime} n^{\prime}}, \omega^{\mu^{\prime}}\right]=-\frac{i}{2}\left(\gamma_{m^{\prime} n^{\prime}} \omega\right)^{\mu^{\prime}} \\
& {\left[d_{\mu}, p_{n}\right]=2\left(\gamma_{n} \omega\right)_{\mu} \quad\left[d_{\mu^{\prime}}, p_{n^{\prime}}\right]=-2\left(\gamma_{n^{\prime}} \omega\right)_{\mu^{\prime}}} \\
& {\left[d_{\mu}, \boldsymbol{F}^{\nu \nu^{\prime}}\right]=-2 \delta_{\mu}^{\nu} \omega^{\nu^{\prime}} \quad\left[d_{\mu^{\prime}}, \mathbf{F}^{\nu \nu^{\prime}}\right]=2 \delta_{\mu^{\prime}}^{\nu^{\prime}} \omega^{\nu}} \\
& \operatorname{dim} 2:\left[s_{m n}, \sigma^{l k}\right]=-i \delta_{[m}^{[k} \sigma_{n]}^{l]} \quad\left[s_{m^{\prime} n^{\prime}}, \sigma^{\left.l^{\prime} k^{\prime}\right]}=i \delta_{\left[m^{\prime}\right.}^{\left[k^{\prime}\right.} \sigma_{n^{\prime}} l^{\prime}\right] \\
& \left\{d_{\mu}, \omega^{\nu}\right\}=-\frac{i}{4} \sigma^{m n}\left(\gamma_{m n}\right)^{\nu}{ }_{\mu}\left\{d_{\mu^{\prime}}, \omega^{\nu^{\prime}}\right\}=\frac{i}{4} \sigma^{m^{\prime} n^{\prime}}\left(\gamma_{m^{\prime} n^{\prime}}\right)^{n^{\prime}} u_{\mu^{\prime}} \\
& {\left[p_{m}, p_{n}\right]=i \sigma_{m n} \quad\left[p_{m^{\prime}}, p_{n^{\prime}}\right]=-i \sigma_{m^{\prime} n^{\prime}}} \\
& {\left[\mathrm{F}^{\mu \nu^{\prime}}, \Upsilon_{\nu \mu^{\prime}}\right]=\frac{i}{4}\left(\delta_{\nu}^{\mu} \sigma^{m^{\prime} n^{\prime}}\left(\gamma_{m^{\prime} n^{\prime}}\right)_{\mu^{\prime}}^{\nu^{\prime}}+\delta_{\mu^{\prime}}^{\nu^{\prime}} \sigma^{m n}\left(\gamma_{m n}\right)^{\mu}{ }_{\nu}\right)}
\end{aligned}
$$

\subsection{Central extension of affine type II super-Poincaré algebra}

An affine extension of (2.2) and (2.3) is presented in this subsection. Generators of the affine Lie algebra are classified by the canonical dimensions as:

\begin{tabular}{|l|c|c|c|c|c|}
\hline $\operatorname{dim}$. & 0 & $\frac{1}{2}$ & 1 & $\frac{3}{2}$ & 2 \\
$\stackrel{\triangleright}{\underline{M}}$ & $S_{m n}, S_{m^{\prime} n^{\prime}}$ & $D_{\mu}, D_{\mu^{\prime}}$ & $\Upsilon_{\mu \nu^{\prime}}, P_{m}, P_{m^{\prime}}, \mathrm{F}^{\mu \nu^{\prime}}$ & $\Omega^{\mu}, \Omega^{\mu^{\prime}}$ & $\Sigma^{m n}, \Sigma^{m^{\prime} n^{\prime}}$ \\
\hline
\end{tabular}

They satisfy

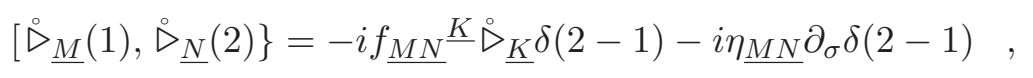

where the structure constant $f_{\underline{M N}} \underline{K}$ is given by (2.2) and (2.3). The nondegenerate metric $\eta_{M N}$ is given as:

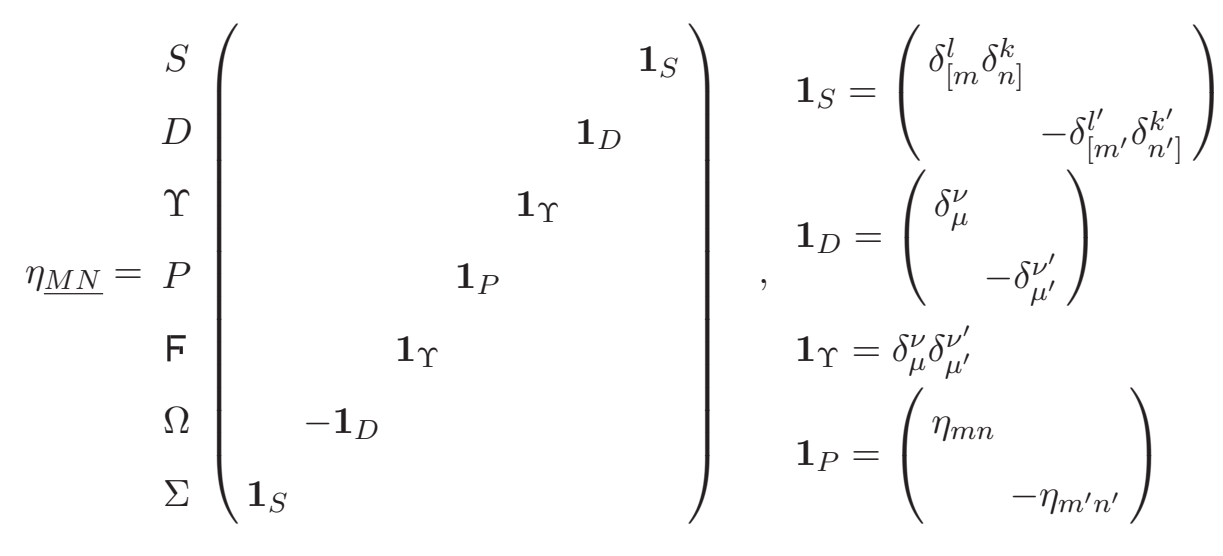


The affine nondegenerate super-Poincaré algebra in components is given by

$$
\begin{aligned}
& \operatorname{dim} 0: {\left[S_{m n}(1), S_{l k}(2)\right]=-i \eta_{[k \mid[m} S_{n] \mid l]} \delta(2-1) } \\
& \operatorname{dim} \frac{1}{2}: {\left[S_{m n}(1), D_{\mu}(2)\right]=-\frac{i}{2}\left(D \gamma_{m n}\right)_{\mu} \delta(2-1) } \\
& \operatorname{dim} 1: {\left[S_{m n}(1), P_{l}(2)\right]=-i P_{[m} \eta_{n] l} \delta(2-1) } \\
&\left\{D_{\mu}(1), D_{\nu}(2)\right\}=2 P_{m} \gamma^{m}{ }_{\mu \nu} \delta(2-1) \\
& \operatorname{dim} \frac{3}{2}:\left[S_{m n}(1), \Omega^{\mu}(2)\right]=\frac{i}{2}\left(\gamma_{m n} \Omega\right)^{\mu} \delta(2-1) \\
& {\left[D_{\mu}(1), P_{n}(2)\right]=2\left(\gamma_{n} \Omega\right)_{\mu} \delta(2-1) } \\
&\left.\operatorname{dim} 2:\left[S_{m n}(1), \Sigma^{l k}(2)\right]=-i \delta_{[m}^{[k} \Sigma_{n]}\right]^{l]} \delta(2-1)+i \delta_{[m}^{l} \delta_{n]}^{k} \partial_{\sigma} \delta(2-1) \\
&\left\{D_{\mu}(1), \Omega^{\nu}(2)\right\}=-\frac{i}{4} \Sigma^{m n}\left(\gamma_{m n}\right)^{\nu}{ }_{\mu} \delta(2-1)+i \delta_{\mu}^{\nu} \partial_{\sigma} \delta(2-1) \\
& {\left[P_{m}(1), P_{n}(2)\right]=i \Sigma_{m n} \delta(2-1)+i \eta_{m n} \partial_{\sigma} \delta(2-1) }
\end{aligned}
$$

Commutators with dimension greater than 2 are zero. The right currents $\left(S^{\prime}, D^{\prime}, P^{\prime}, \Omega^{\prime}, \Sigma^{\prime}\right)$ satisfy the same algebra with opposite sign in the right hand sides.

Algebras involving the R-R charges $\Upsilon_{\mu \mu^{\prime}}$ and $\mathrm{F}^{\mu \mu^{\prime}}$ are the followings:

$$
\begin{aligned}
& \operatorname{dim} 1:\left[S_{m n}(1), \mathrm{F}^{\mu \nu^{\prime}}(2)\right]=\frac{i}{2}\left(\gamma_{m n}\right)^{\mu}{ }_{\nu} \mathrm{F}^{\nu \nu^{\prime}} \delta(2-1)
\end{aligned}
$$

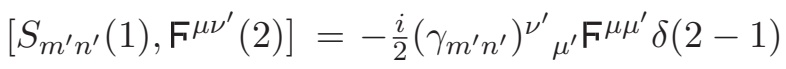

$$
\begin{aligned}
& {\left[S_{m n}(1), \Upsilon_{\mu \nu^{\prime}}(2)\right]=-\frac{i}{2} \Upsilon_{\nu \nu^{\prime}}\left(\gamma_{m n}\right)^{\nu}{ }_{\mu} \delta(2-1)} \\
& {\left[S_{m^{\prime} n^{\prime}}(1), \Upsilon_{\mu \nu^{\prime}}(2)\right]=\frac{i}{2} \Upsilon_{\mu \mu^{\prime}}\left(\gamma_{m^{\prime} n^{\prime}}\right)^{\mu^{\prime}}{ }_{\nu^{\prime}} \delta(2-1)} \\
& \left\{D_{\mu}(1), D_{\nu^{\prime}}(2)\right\} \quad=2 \Upsilon_{\mu \nu^{\prime}} \delta(2-1) \\
& \operatorname{dim} \frac{3}{2}:\left[D_{\mu}(1), \mathrm{F}^{\nu \nu^{\prime}}(2)\right]=-2 \delta_{\mu}^{\nu} \Omega^{\nu^{\prime}} \delta(2-1) \\
& {\left[D_{\mu^{\prime}}(1), \boldsymbol{F}^{\nu \nu^{\prime}}(2)\right]=2 \delta_{\mu^{\prime}}^{\nu^{\prime}} \Omega^{\nu} \delta(2-1)} \\
& \operatorname{dim} 2:\left[\mathrm{F}^{\mu \nu^{\prime}}(1), \Upsilon_{\nu \mu^{\prime}}(2)\right]=\frac{i}{4}\left(\delta_{\nu}^{\mu} \Sigma^{m^{\prime} n^{\prime}}\left(\gamma_{m^{\prime} n^{\prime}}\right)_{\mu^{\nu^{\prime}}}+\delta_{\mu^{\prime}}^{\nu^{\prime}} \Sigma^{m n}\left(\gamma_{m n}\right)^{\mu}{ }_{\nu}\right) \delta(2-1) \\
& +i \delta_{\nu}^{\mu} \delta_{\mu^{\prime}}^{\nu^{\prime}} \partial_{\sigma}(2-1)
\end{aligned}
$$

Commutators with dimension greater than 2 are zero.

\section{$3 \kappa$-symmetric Virasoro constraints}

As the Virasoro constraint restricts string backgrounds, the $\kappa$-symmetry constraint together with the Virasoro constraint restrict superstring backgrounds [39]. The $\kappa$-symmetry at the first quantized level guarantees the number of degrees of freedom of fermionic coordinates, while the $\kappa$-symmetric Virasoro constraint guarantees the physical degrees of freedom of 
the background fields. It was also shown that the $\kappa$-symmetric Virasoro algebra restricts torsions in a superspace $[40,41]$. This restriction was presented for the manifestly T-dual type II superspace [18]. In this section consistency of the $\kappa$-symmetry is examined from the fermionic constraints of the Green-Schwarz superstring. Then $\kappa$-symmetric Virasoro constraints are obtained.

\section{$3.1 \kappa$-symmetry}

In the Green-Schwarz formalism fermionic constraints, $D_{\mu}=\left(D_{\mu}, D_{\mu^{\prime}}\right)=0$, are mixed constraints of first class and second class, where the first class constraints generate the $\kappa$-symmetry. In order to preserve this structure an anticommutator between two fermionic constraints must have zero determinant,

$$
\left\{D_{\underline{\mu}}, D_{\underline{\nu}}\right\}=\Xi_{\underline{\mu \nu}}, \quad \Xi_{\underline{\mu \nu}}=2\left(\begin{array}{cc}
\not P_{\mu \nu} & \Upsilon_{\mu \nu^{\prime}} \\
\Upsilon_{\nu \mu^{\prime}} & -\not P_{\mu^{\prime} \nu^{\prime}}
\end{array}\right), \quad \operatorname{det} \Xi_{\underline{\mu \nu}}=0
$$

with $\not P=P_{m} \gamma^{m}$. $\Xi_{\underline{\mu \nu}}$ must have rank 16 and it has 16 independent zero eigen vectors. Zero eigen matrix of $\bar{\Xi}_{\mu \nu}$ is chosen with a constant $\alpha$ as

$$
\bar{\Xi}^{\mu \nu}=\left(\begin{array}{cc}
P^{\mu \nu} & \alpha \bar{F}^{\mu \nu^{\prime}} \\
\alpha \bar{F}^{\nu \mu^{\prime}} & P^{\mu^{\prime} \nu^{\prime}}
\end{array}\right)
$$

then it satisfies

$$
\Xi_{\mu \nu} \bar{\Xi} \underline{\underline{\nu}} \approx 0 \Leftrightarrow\left\{\begin{array}{l}
P_{m} P^{m}+\frac{\alpha}{16} \operatorname{tr} \Upsilon \mathrm{F}=0 \\
P_{m^{\prime}} P^{m^{\prime}}-\frac{\alpha}{16} \operatorname{tr} \Upsilon \mathrm{F}=0 \\
\left.\Upsilon_{\mu \nu^{\prime}} \mathrm{F}^{\nu \nu^{\prime}}\right|_{\operatorname{trls}}=\left.\Upsilon_{\mu \mu^{\prime}} \mathrm{F}^{\mu \nu^{\prime}}\right|_{\text {trls }}=0 \\
\Upsilon_{\mu \mu^{\prime}} P^{\mu^{\prime} \nu^{\prime}}+\alpha \not P_{\mu \nu} \mathrm{F}^{\nu \nu^{\prime}}=0 \\
P^{\mu \nu} \Upsilon_{\nu \nu^{\prime}}-\alpha \mathrm{F}^{\mu \mu^{\prime}} \not_{\mu^{\prime} \nu^{\prime}}=0
\end{array}\right.
$$

The first and second lines of (3.3) are Klein-Gordon operators and the last two lines are Dirac-Kähler operators. The term tr $\Upsilon F$ becomes BPS mass for D-branes where $\Upsilon$ and $F$ take D-brane volume forms [36-38]. The $\kappa$-symmetry constraints are obtained as

$$
\mathcal{B}_{0}^{\underline{\mu}}=D_{\underline{\nu}} \overline{\Xi^{\nu}} \underline{\mu}=\left\{\begin{array}{l}
\mathcal{B}_{0}^{\mu}=D_{\nu} \not^{\nu \mu}+\alpha D_{\nu^{\prime}} \mathrm{F}^{\mu \nu^{\prime}}=0 \\
\mathcal{B}_{0}^{\mu^{\prime}}=D_{\nu^{\prime}} P^{\nu^{\prime} \mu^{\prime}}+\alpha D_{\nu} \mathrm{F}^{\nu \mu^{\prime}}=0
\end{array}\right.
$$

so that they are first class

$$
\left\{\mathcal{B}_{0}^{\mu}, \mathcal{B}_{0}^{\underline{\nu}}\right\}=2 \overline{\bar{\Xi}} \underline{\mu \rho} \Xi_{\underline{\rho \lambda}} \bar{\Xi} \underline{\lambda \nu}+(D, D D \text { constraints }) \approx 0 .
$$


In this paper we focus on background solutions which couple to superstrings not Dbranes, so we impose an additional constraint

$$
\Upsilon_{\mu \nu^{\prime}}=0
$$

Consistency with (3.3) and (3.6), $\{\Upsilon, \Xi \bar{\Xi}\} \approx 0$, leads to $\alpha=0$. This gives massless conditions:

$$
\Xi_{\underline{\mu \nu}} \bar{\Xi} \underline{\underline{ }} \underline{ } \approx 0 \Leftrightarrow\left\{\begin{array}{l}
P_{m} P^{m}=0 \\
P_{m^{\prime}} P^{m^{\prime}}=0
\end{array}\right.
$$

Although it reduces to the same algebra with the $\kappa$-symmetric Virasoro algebra without central extension, $\mathrm{F}^{\mu \nu^{\prime}}$ is necessary to introduce the R-R gauge field.

\section{$3.2 \kappa$-symmetric Virasoro constraints}

The $\sigma$-diffeomorphism constraint is given by

$$
\begin{aligned}
\mathcal{H}_{\sigma} & =\frac{1}{2} \stackrel{\triangleright}{\triangleright}_{\underline{M}} \eta \underline{\underline{M N}} \stackrel{\circ}{\triangleright}_{\underline{N}} \\
& =\frac{1}{2} P_{m} P^{m}-\frac{1}{2} P_{m^{\prime}} P^{m^{\prime}}+\Omega^{\mu} D_{\mu}-\Omega^{\mu^{\prime}} D_{\mu^{\prime}}+\frac{1}{2} \Sigma^{m n} S_{m n}-\frac{1}{2} \Sigma^{m^{\prime} n^{\prime}} S_{m^{\prime} n^{\prime}}+\mathrm{F}^{\mu \nu^{\prime}} \Upsilon_{\mu \nu^{\prime}}
\end{aligned}
$$

satisfying

$$
\left[\mathcal{H}_{\sigma}(\sigma), \mathcal{H}_{\sigma}\left(\sigma^{\prime}\right)\right]=-i\left(2 \mathcal{H}_{\sigma}(\sigma) \partial_{\sigma} \delta\left(\sigma-\sigma^{\prime}\right)+\partial_{\sigma} \mathcal{H}_{\sigma} \delta\left(\sigma-\sigma^{\prime}\right)\right)
$$

The commutator with $\mathcal{H}_{\sigma}$ gives $\sigma$ derivatives of operators, for example $\left[\mathcal{O}(\sigma), i \int \mathcal{H}_{\sigma}\right]=$ $\partial_{\sigma} \mathcal{O}(\sigma)$. The $\tau$-diffeomorphism constraint is given by

$$
\begin{aligned}
& \mathcal{H}_{\tau}=\frac{1}{2} \stackrel{\circ}{\triangleright}_{\underline{M}} \hat{\eta}^{\underline{M N}} \stackrel{\circ}{\triangleright}_{\underline{N}} \\
& =\frac{1}{2} P_{m} P^{m}+\frac{1}{2} P_{m^{\prime}} P^{m^{\prime}}+\Omega^{\mu} D_{\mu}+\Omega^{\mu^{\prime}} D_{\mu^{\prime}}+\frac{1}{2} \Sigma^{m n} S_{m n}+\frac{1}{2} \Sigma^{m^{\prime} n^{\prime}} S_{m^{\prime} n^{\prime}}
\end{aligned}
$$

where a metric $\hat{\eta}_{\underline{M N}}$ is defined as:

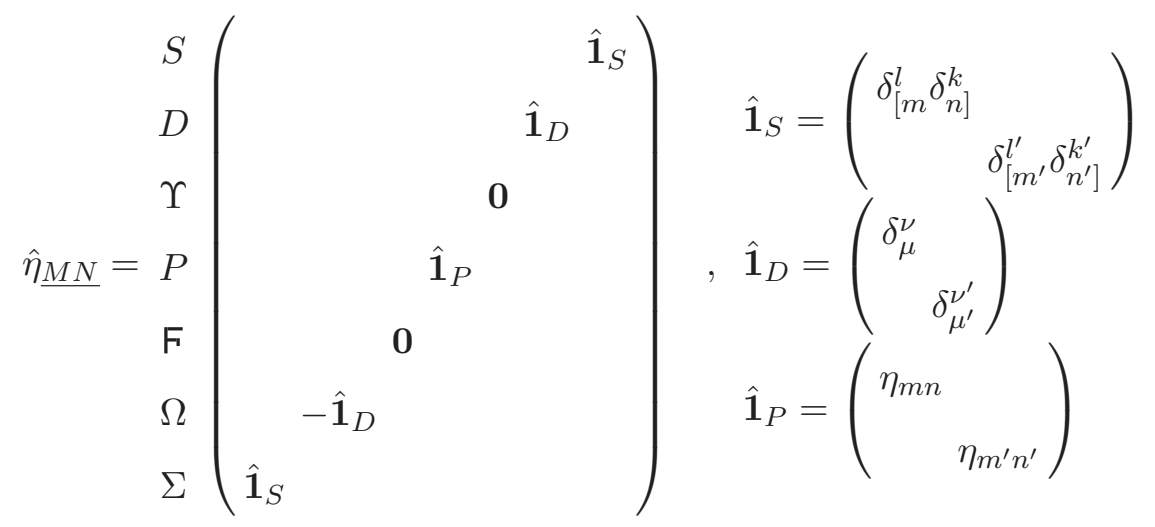


$\mathcal{H}_{\sigma}=\mathcal{H}_{\tau}=0$ are essentially massless constraints in two spaces given in (3.7) if we use first and second class constraints, $D_{\mu}=D_{\mu^{\prime}}=0$ and $S_{m n}=S_{m^{\prime} n^{\prime}}=\Upsilon_{\mu \nu^{\prime}}=0$. They satisfy the following Virasoro algebra

$$
\begin{aligned}
{\left[\mathcal{H}_{\sigma}(1), \mathcal{H}_{\tau}(2)\right] } & =i\left(\mathcal{H}_{\tau}(1)+\mathcal{H}_{\tau}(2)\right) \partial_{\sigma} \delta(2-1) \\
{\left[\mathcal{H}_{\tau}(1), \mathcal{H}_{\tau}(2)\right] } & =i\left\{\left(\mathcal{H}_{\sigma}-\operatorname{tr} \boldsymbol{F} \Upsilon\right)(1)+\left(\mathcal{H}_{\sigma}-\operatorname{tr} F \Upsilon\right)(2)\right\} \partial_{\sigma} \delta(2-1) \\
{\left[\mathcal{H}_{\sigma}(1), \Upsilon_{\mu \nu^{\prime}}(2)\right] } & =i \Upsilon_{\mu \nu^{\prime}}(1) \partial_{\sigma} \delta(2-1)+\frac{i}{4}\left(\delta_{\mu}^{\rho} \mathcal{H}^{\rho^{\prime}}{ }_{\nu^{\prime}}+\delta_{\nu^{\prime}}^{\rho^{\prime}}{ }^{\rho}{ }_{\mu}\right) \Upsilon_{\rho \rho^{\prime}} \delta(2-1) \\
{\left[\mathcal{H}_{\tau}(1), \Upsilon_{\mu \nu^{\prime}}(2)\right] } & =0
\end{aligned}
$$

with $\mathbb{Z}=\Sigma_{m n} \gamma^{m n}$. Other constraints except $\mathcal{H}_{\sigma}$ do not include $\mathrm{F}^{\mu \nu^{\prime}}$ so they commute with $\Upsilon$, such as $\Upsilon_{\mu \nu^{\prime}}=0$ and $S_{m n}=S_{m^{\prime} n^{\prime}}=0$.

A consistent set of $\kappa$-symmetric Virasoro constraints are

$$
\left(\mathcal{H}_{\tau}, \mathcal{H}_{\sigma}, \mathcal{B}^{\mu}, \mathcal{B}^{\mu^{\prime}}, S_{m n}, S_{m^{\prime} n^{\prime}}, \Upsilon_{\mu \nu^{\prime}}, \mathcal{C}_{\mu \nu}, \mathcal{C}_{\mu^{\prime} \nu^{\prime}}, \mathcal{D}_{m}, \mathcal{D}_{m^{\prime}}\right)=0
$$

$\mathcal{C D}$ constraints are constructed by bilinears of $D_{\mu}$ and $D_{\mu^{\prime}} \cdot \mathcal{A B C D}$ constraints make a closed algebra as same as the one without central extension up to the $\Upsilon=0$ constraints given in [18]. $\mathcal{A B C D}$ constraints are extended to curved backgrounds as shown in [18]. The $\kappa$-symmetric Virasoro algebras for flat left and flat right with $\Upsilon$ and $F$ are given as

$$
\begin{aligned}
\mathcal{A} & =\frac{1}{2} P_{m} P^{m}+\Omega^{\mu} D_{\mu}+\frac{1}{2} \Sigma^{m n} S_{m n}+\frac{1}{2} F^{\mu \nu^{\prime}} \Upsilon_{\mu \nu^{\prime}} \\
\mathcal{B}^{\mu} & =D_{\nu} P^{\nu \mu}-i S_{m n}\left(\gamma^{m n} \Omega\right)^{\mu} \\
\mathcal{A}^{\prime} & =\frac{1}{2} P_{m^{\prime}} P^{m^{\prime}}+\Omega^{\mu^{\prime}} D_{\mu^{\prime}}+\frac{1}{2} \Sigma^{m^{\prime} n^{\prime}} S_{m^{\prime} n^{\prime}}-\frac{1}{2} F^{\mu \nu^{\prime}} \Upsilon_{\mu \nu^{\prime}} \\
\mathcal{B}^{\mu^{\prime}} & =D_{\nu^{\prime}} P^{\nu^{\prime} \mu^{\prime}}-i S_{m^{\prime} n^{\prime}}\left(\gamma^{m^{\prime} n^{\prime}} \Omega\right)^{\mu^{\prime}}
\end{aligned}
$$

\section{Torsion and vielbein}

Now we examine superstring backgrounds by introducing the vielbein field. Simplification occurs by the central extension of $\Upsilon_{\mu \nu^{\prime}}$ among several results following our previous paper [18]. The vielbein field includes the R-R gauge fields as well as $\mathrm{R}-\mathrm{R}$ field strengths. Gauge transformation rules and solutions of torsion constraints are presented. We identify type II supergravity fields to vielbein field components $E_{\underline{A}} \underline{\underline{M}}$.

\subsection{Torsion constraints}

Covariant derivatives in curved backgrounds are written in terms of vielbein fields as

$$
\begin{aligned}
\triangleright_{\underline{A}} & =E_{\underline{A}}^{\underline{M}}\left(Z^{\underline{N}}\right) \stackrel{\triangleright}{\triangleright}_{\underline{M}} \\
{\left[\triangleright_{\underline{A}}, \triangleright_{\underline{B}}\right\} } & =-i \underline{T}_{\underline{A B}}^{\underline{C}} \triangleright_{\underline{C}} \delta(2-1)-i \eta_{\underline{A B}} \partial_{\sigma} \delta(2-1) .
\end{aligned}
$$

The manifestly T-dual formulation allows to impose an orthonormal condition on the vielbein field. It results the second term of the right hand side of (4.1) as

$$
E_{\underline{A}}^{\underline{M}} \eta_{\underline{M N}} E_{\underline{B}}^{\underline{N}}=\eta_{\underline{A B}}, \quad \eta_{\underline{M N}}=\eta_{\underline{A B}} .
$$


Torsions and Bianchi identities with lower indices are totally graded antisymmetric

$$
\begin{gathered}
T_{\underline{A B C}}=T_{\underline{A B}} \underline{D} \eta_{\underline{D C}}=\frac{1}{3 !} T_{[\underline{A B C})}=\frac{1}{2}\left(D_{[\underline{A}} E_{\underline{B}} \underline{M}\right) E_{\underline{C}) \underline{M}}+E_{\underline{A}} \underline{M} E_{\underline{B}} \underline{\underline{N}} E_{\underline{C}} \underline{\underline{L}} f_{\underline{M N L}} \\
\mathcal{I}_{\underline{A B C D}}=\frac{1}{4 !} \mathcal{I}_{[\underline{A B C D})}=D_{[\underline{A}} T_{\underline{B C D})}+\frac{3}{4} T_{[\underline{A B}} \underline{\underline{E}} T_{\underline{C D}) \underline{E}}=0
\end{gathered}
$$

with $D_{\underline{A}}=E_{\underline{A}} \underline{\underline{M}} D_{\underline{M}}$ and $f_{\underline{M N L}}=f_{\underline{M N}} \underline{\underline{K}} \eta_{\underline{K L}}$ is the structure constant in (2.5). Graded antisymmetrization is denoted as $\mathcal{O}_{[A B)}=\overline{\mathcal{O}_{A B}}-(-)^{A B} \mathcal{O}_{B A}$.

The Bianchi identity and the consistency of the $\kappa$-symmetry determine a set of torsion constraints. Indices are abbreviated as $V_{a}=V_{P_{a}}, V_{\underline{a}}=\left(V_{P_{a}}, V_{P_{a^{\prime}}}\right), V_{\alpha}=V_{D_{\alpha}}, V_{\underline{\alpha}}=$ $\left(V_{D_{\alpha}}, V_{D_{\alpha^{\prime}}}\right)$. We impose torsion constraints for Lorentz indices as

$$
T_{\underline{S A B}}=f_{\underline{S A B}} .
$$

Then torsions with two $P_{a}$ 's indices, $T_{a b} \underline{\underline{M}}$, are absorbed into $E_{\Sigma} \underline{\underline{M}}$ as

$$
\left[\triangleright_{a}, \triangleright_{b}\right]=T_{a b} \underline{A}^{\underline{A}} \triangleright_{A} \rightarrow f_{a b}{ }^{\Sigma} \triangleright_{\Sigma}=f_{a b S} E_{\Sigma}{ }^{\underline{M}} Z_{\underline{M}} .
$$

For superspaces with fermionic constraints, most of torsions with dimension lower than 1 are structure constants or zero. A supercurvature $T_{\alpha \beta \Sigma}$ is absorbed into vielbein $E_{c \Sigma}$ as $f_{\alpha \beta}{ }^{c} E_{c \Sigma}$. The supercurvature $R_{\alpha \beta^{\prime}}{ }^{c d}=T_{\alpha \beta \Sigma}$, which was nonzero for AdS space, is absorbed into vielbein after introducing $\Upsilon_{\mu \nu^{\prime}}$ as

$$
\left\{\triangleright_{\alpha}, \triangleright_{\beta^{\prime}}\right\}=T_{\alpha \beta^{\prime} \Sigma} S \rightarrow f_{\alpha \beta^{\prime}}{ }^{\Upsilon} E_{\Upsilon \Sigma} S .
$$

A dimension 1 torsion $T_{\alpha^{\prime} a}{ }^{\gamma}$, which was nonzero without $\Upsilon_{\mu \nu^{\prime}}$, becomes zero from the Bianchi identity $\mathcal{I}_{\alpha \alpha^{\prime} a b}=0$ in (4.3) with help of $T_{a b \Upsilon}=0$ in (4.5). 
Trivial and non-trivial torsions are listed as follows:

\begin{tabular}{|c|c|c|}
\hline $\operatorname{dim}$ & zero & nonzero \\
\hline$-1 / 2$ & $T_{\alpha \beta \gamma}, T_{\alpha \beta \gamma^{\prime}}$ & NONE \\
\hline 0 & 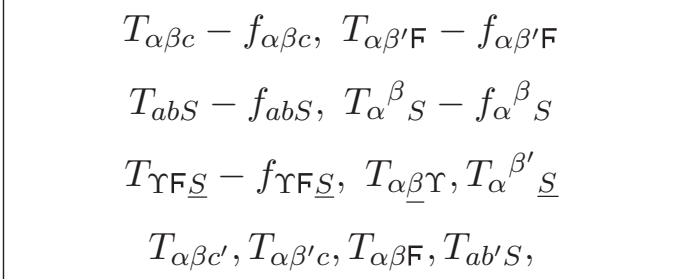 & NONE \\
\hline $1 / 2$ & $\begin{array}{c}T_{\alpha \underline{\underline{\beta}}} \underline{\underline{\gamma}}, T_{\alpha b \underline{c}}, T_{\alpha b^{\prime} c^{\prime}}, T_{\alpha \underline{b} \Upsilon}, T_{\alpha \underline{b} \mathfrak{F}} \\
, T_{\alpha \Upsilon \Upsilon}, T_{\alpha \Upsilon F}, T_{\alpha \mathrm{FF}}\end{array}$ & NONE \\
\hline 1 & $\begin{array}{c}T_{\alpha \underline{\underline{b}}} \underline{\underline{\gamma}}, T_{\alpha \Upsilon} \underline{\gamma}, T_{\alpha \mathrm{F}^{-}}, T_{\alpha \underline{\beta} \underline{\underline{\Sigma}}}, \\
T_{a b \underline{\underline{c}}}, T_{a \underline{b} \Upsilon}, T_{a \underline{b} \mathrm{~F}}, T_{a \Upsilon \Upsilon}, T_{a \Upsilon \mathrm{F}}, T_{a \mathrm{FF}} \\
T_{\Upsilon \Upsilon \Upsilon}, T_{\Upsilon \Upsilon \mathrm{F}}, T_{\Upsilon \mathrm{FF}}, T_{\mathrm{FF} F}\end{array}$ & NONE \\
\hline $3 / 2$ & $T_{\alpha} \underline{\underline{\beta \gamma}}, T_{a b^{\underline{\gamma}}}, T_{\alpha \underline{b \Sigma}}, T_{\alpha \mathrm{F} \Sigma}, T_{a \mathrm{~F}^{\beta}}, T_{a \Upsilon^{\beta^{\prime}}}, T_{\Upsilon \mathrm{F}} \underline{\beta}$ & $\begin{array}{l}T_{\alpha^{\prime} b \Sigma} \sim T_{\alpha \Upsilon \Sigma} \sim T_{a \Upsilon}{ }^{\alpha} \\
T_{a b^{\prime}}{ }^{\beta} \sim T_{\alpha b^{\prime} \Sigma} \sim T_{\alpha^{\prime} \Upsilon \Sigma} \\
\sim T_{\Upsilon \Upsilon^{\beta}} \sim T_{a F^{F^{\prime}}} \sim T_{\mathrm{FF}}{ }^{\beta}\end{array}$ \\
\hline 2 & $T_{\alpha}^{\beta^{\prime}}{ }_{\Sigma^{\prime}}, T_{\Upsilon}^{\alpha \beta}, T_{a \Upsilon \Sigma}, T_{\mathrm{FF} \Sigma}$ & $\begin{array}{c}T_{a b \Sigma} \sim T_{\alpha}^{\beta} \Sigma \sim T_{a}^{\alpha \beta} \\
T_{a^{\prime} b^{\prime} \Sigma} \sim T_{\alpha}^{\beta}{ }_{\Sigma^{\prime}} \sim T_{\Upsilon}{ }^{\alpha \beta^{\prime}} \\
T_{a}{ }^{\beta^{\prime}} \sim T_{\alpha}^{\beta^{\prime}} \sim T_{a^{\prime} \Upsilon \mathrm{F}} \\
T_{a b^{\prime} \Sigma} \sim T_{a}{ }^{\alpha^{\prime} \beta^{\prime}} \\
T_{a \mathrm{~F} \Sigma} \sim T_{\mathrm{F}}{ }^{\alpha \beta} \\
T_{\mathrm{F}}{ }^{\beta^{\prime}}, T_{\Upsilon \Upsilon \Sigma}, T_{\Upsilon F \Sigma}\end{array}$ \\
\hline
\end{tabular}

The same relation holds for primed indices. This formalism allows a simple treatment where all torsions with dimension 1 and less are structure constants otherwise zero.

\subsection{Vielbein}

Vectors in the space $\hat{\Lambda}_{i}=\Lambda_{i} \underline{\underline{M}} \stackrel{\circ}{\underline{M}}_{\underline{M}}$ satisfy the following supersymmetric Lie bracket in the manifestly T-dual formulation which is obtained from zero-mode of (2.5)

$$
\begin{aligned}
{\left[\hat{\Lambda}_{1}, \hat{\Lambda}_{2}\right]_{\mathrm{T}} } & =-i \hat{\Lambda}_{12} \\
\Lambda_{12} \underline{\underline{M}} & \left.=\Lambda_{[1} \underline{\underline{N}}\left(D_{\underline{N}} \Lambda_{2}\right]^{\underline{M}}\right)-\frac{1}{2} \Lambda_{[1} \underline{\underline{N}}\left(D^{\underline{M}} \Lambda_{2] \underline{N}}\right)+\Lambda_{1} \underline{\underline{N}} \Lambda_{2} \underline{\underline{L}} f_{\underline{N L}} \underline{\underline{M}}+k \Lambda_{(1} \underline{N}\left(D^{\underline{M}} \Lambda_{2) \underline{N}}\right)
\end{aligned}
$$

with arbitrary number $k$ which arises from an ambiguity of $\partial_{\sigma} \delta(\sigma)$. The vielbein field $\hat{E}_{\underline{A}}=E_{\underline{A}} \underline{\underline{M}} \stackrel{\circ}{ }_{\underline{M}}$ has gauge symmetry generated by the manifestly T-dual bracket given in 
(4.8) with $k=-1 / 2$.

$$
\begin{aligned}
\delta_{\Lambda} \hat{E}_{\underline{A}} & =i\left[\hat{E}_{\underline{A}}, \hat{\Lambda}\right]_{\mathrm{T}} \\
\delta_{\Lambda} E_{\underline{A M}} & =D_{\underline{A}} \Lambda_{\underline{M}}-\Lambda^{\underline{N}}\left(D_{\underline{N}} E_{\underline{A M}}\right)-E_{\underline{A}} \underline{\underline{N}}\left(D_{\underline{M}} \Lambda_{\underline{N}}\right)+E_{\underline{A}} \underline{\underline{N}} \Lambda \underline{\underline{L}} f_{\underline{N L M}}
\end{aligned}
$$

with $\Lambda_{\underline{M}}=\Lambda^{\underline{N}} \eta_{\underline{N M}}$.

In order to identify components of the supervielbein field with the field content of type II supergravity, dimensional reduction of two Lorentz degrees of freedom into one local Lorentz degree of freedom is realized as $S_{m n}+\left(-S_{m^{\prime} n^{\prime}}\right) \rightarrow S_{\mathrm{mn}}$. Momentum/winding coordinates, $p_{\mathrm{m}} / \partial_{\sigma} x^{\mathrm{m}}$, related to the left/right coordinates $P_{m}, P_{m^{\prime}}$ as $V_{\mathrm{m}}=\left(V_{m}+V_{m^{\prime}}\right) / 2$ and $V^{\mathrm{m}}=\left(V_{m}-V_{m^{\prime}}\right) / 2$. The vielbein $E_{\underline{\mathrm{a}}} \underline{\mathrm{m}}$ has the following form

$$
\begin{aligned}
& E_{\underline{\mathrm{a}}}^{\underline{\mathrm{m}}}=\left(\begin{array}{cc}
E_{\mathrm{a}}^{\mathrm{m}} & E_{\mathrm{am}} \\
E^{\mathrm{am}} & E^{\mathrm{a}}{ }_{\mathrm{m}}
\end{array}\right)=\left(\begin{array}{cc}
e_{\mathrm{a}}{ }^{\mathrm{m}} & e_{\mathrm{a}}{ }^{\mathrm{n}} B_{\mathrm{nm}} \\
0 & e_{\mathrm{m}}{ }^{\mathrm{a}}
\end{array}\right) \\
& \Rightarrow E_{\underline{\mathrm{a}}}{ }_{\underline{\mathrm{m}}} \eta_{\underline{\mathrm{mn}}} E_{\underline{\mathrm{b}}}^{\underline{\mathrm{n}}}=\eta_{\underline{\mathrm{ab}}}, \quad E_{\underline{\mathrm{a}}} \eta^{\underline{\mathrm{m}}} E_{\underline{\mathrm{b}}}^{\underline{\mathrm{n}}}=\eta^{\underline{\mathrm{mn}}}, \quad \eta_{\underline{\mathrm{ab}}}=\eta_{\underline{\mathrm{mn}}}=\left(\begin{array}{ll}
0 & 1 \\
1 & 0
\end{array}\right) \\
& E_{\underline{\mathrm{a}}} \underline{\underline{\mathrm{m}}} \hat{\eta}^{\underline{\mathrm{ab}}} E_{\underline{\mathrm{b}}} \underline{\underline{\mathrm{n}}}=\left(\begin{array}{cc}
G^{\mathrm{mn}} & G^{\mathrm{ml}} B_{\mathrm{ln}} \\
B_{\mathrm{ml}} G^{\mathrm{lb}} & G_{\mathrm{mn}}-B_{\mathrm{ml}} G^{\mathrm{lk}} B_{\mathrm{kn}}
\end{array}\right) \quad, \quad \hat{\eta} \underline{\underline{\mathrm{ab}}}=\left(\begin{array}{ll}
1 & 0 \\
0 & 1
\end{array}\right) .
\end{aligned}
$$

The general coordinate transformations of $e_{\mathrm{a}}{ }^{\mathrm{m}}$ and $B_{\mathrm{mn}}$ are given by $\delta_{\xi} \hat{E}=i\left[\hat{E}, \xi^{\underline{m}}{\stackrel{\circ}{D_{m}}}\right]_{\mathrm{T}}$ as

$$
\begin{aligned}
\delta_{\xi} e_{\mathrm{a}}{ }^{\mathrm{m}} & =D_{\mathrm{a}} \xi^{\mathrm{m}}-\xi^{\underline{\underline{n}}} \partial_{\underline{n}} e_{\mathrm{a}}{ }^{\mathrm{m}}-e_{\mathrm{a}}{ }^{\underline{\mathrm{n}}} \partial^{\mathrm{m}} \xi_{\underline{\mathrm{n}}}+\omega_{\mathrm{a}}{ }^{\mathrm{ml}} \xi_{1} \\
\delta_{\xi} B_{\mathrm{mn}} & =\partial_{[\mathrm{m}} \xi_{\mathrm{n}]}-\xi^{\underline{l}} \partial_{\underline{l}} B_{\mathrm{mn}}-e_{\left[\mathrm{m}^{-}\right.} \partial_{\mathrm{n}]} \xi_{\underline{l}}
\end{aligned}
$$

The gauge parameters are $\xi^{\underline{m}}=\left(\xi^{\mathrm{m}}, \xi_{\mathrm{m}}\right)$ where $\xi_{\mathrm{m}}$ is the $B$ field gauge parameter. The NS-NS field strength is $\omega_{\mathrm{abc}}=F_{\mathrm{NS} ; \mathrm{abc}}=\frac{1}{2} \partial_{[\mathrm{a}} B_{\mathrm{bc}}$, while $\omega_{\mathrm{bc}}^{\mathrm{a}} \sim e(\partial e) e$ is the usual part. Gravitino and dilatino fields are included in $E_{a \Omega}$ as $e_{\mathrm{m}}{ }^{\mathrm{a}} E_{\mathrm{a}}{ }^{\mu}=\psi_{\mathrm{m}}{ }^{\mu}+\left(\gamma_{\mathrm{m}} \lambda\right)^{\mu}$.

The R-R gauge field is $e_{\mathrm{m}}{ }^{\mathrm{a}} E_{\mathrm{ar}}=C_{\mathrm{RR} ; \mathrm{m}^{\mu \nu^{\prime}}}$, where two spinor indices $C^{\mu \nu^{\prime}}$ are expanded by odd/even number antisymmetric gamma matrices for type IIB/IIA. The gauge transfor-

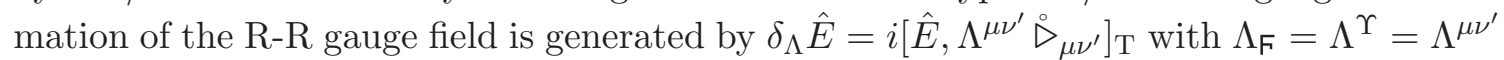
as

$$
\begin{aligned}
\Lambda_{\mathrm{F}} & =\Lambda^{\mu \nu^{\prime}}=\sum_{\mathrm{p}=\text { odd }} \Lambda_{\mathrm{n}_{1} \cdots \mathrm{n}_{\mathrm{p}}}\left(\tau_{i}\right)_{12}\left(\gamma^{\mathrm{n}_{1} \cdots \mathrm{n}_{\mathrm{p}}}\right)^{\alpha \beta} \text { with }\left\{\begin{array}{l}
\tau_{i}=\tau_{1} \text { for } \mathrm{p}=1,5 \\
\tau_{i}=i \tau_{2} \text { for } \mathrm{p}=3
\end{array}\right. \\
e_{\mathrm{m}}{ }^{\mathrm{a}} E_{a \mathrm{~F}} & =C_{\mathrm{RR} ; \mathrm{m}}{ }^{\mu \nu^{\prime}}=\sum_{\mathrm{p}=\text { even }}\left(C_{\mathrm{RR}}\right)_{\mathrm{mn}_{1} \cdots \mathrm{n}_{\mathrm{p}}}\left(\tau_{i}\right)_{12}\left(\gamma^{\mathrm{n}_{1} \cdots \mathrm{n}_{\mathrm{p}}}\right)^{\mu \nu} \\
\delta C_{\mathrm{RR}}{ }_{\mathrm{m}}^{\mu \nu^{\prime}} & =\partial_{\mathrm{m}} \Lambda^{\mu \nu^{\prime}} \Rightarrow \delta C_{\mathrm{RRmn}} \cdots \mathrm{n}_{\mathrm{p}}=\partial_{[\mathrm{m}} \Lambda_{\left.\mathrm{n}_{1} \cdots \mathrm{n}_{\mathrm{p}}\right]} .
\end{aligned}
$$

Local supersymmetry transformations are generated by $\delta_{\varepsilon} \hat{E}=i\left[\hat{E}, \varepsilon^{\underline{\mu}} \stackrel{\circ}{\perp}_{\underline{\mu}}\right]_{\mathrm{T}}$ with parameters $\varepsilon^{\underline{\mu}}=\left(\varepsilon^{\mu}, \varepsilon^{\mu^{\prime}}\right)$ in the first order of fermionic fields as

$$
\delta_{\varepsilon} e_{\mathrm{a}}{ }^{\mathrm{m}}=-B_{\mathrm{a}} \underline{\underline{\mu}} \partial^{\mathrm{m}} \varepsilon^{\underline{\mu}}+\psi_{\mathrm{a}} \underline{\underline{\mu}}\left(\gamma^{\mathrm{m}} \varepsilon\right)_{\underline{\mu}}
$$




$$
\begin{aligned}
& \delta_{\varepsilon} B_{\mathrm{mn}}=-B_{[\mathrm{m} \mid \underline{\mu}} \partial_{\mid \mathrm{n}]} \varepsilon^{\underline{\mu}}+\psi_{[\mathrm{m}}{ }^{\mu}\left(\gamma_{\mathrm{n}]} \varepsilon\right)_{\mu}-\psi_{[\mathrm{m}}{ }^{\mu^{\prime}}\left(\gamma_{\mathrm{n}]} \varepsilon\right)_{\mu^{\prime}} \\
& \delta_{\varepsilon} C_{\mathrm{RRm}}{ }^{\mu \nu^{\prime}}=\frac{1}{2} \psi_{\mathrm{m}}{ }^{(\mu} \varepsilon^{\left.\nu^{\prime}\right)}+\frac{1}{2}\left(\lambda \gamma_{\mathrm{m}}\right)^{(\mu} \varepsilon^{\left.\nu^{\prime}\right)}-\frac{1}{2}\left(E_{\mathrm{F}}{ }^{\mu}\left(\gamma_{\mathrm{m}} \varepsilon\right)_{\mu}-E_{\mathrm{F}}{ }^{\mu^{\prime}}\left(\gamma_{\mathrm{m}} \varepsilon\right)_{\mu^{\prime}}\right) \\
& \delta_{\varepsilon} \psi_{\mathrm{m}}{ }^{\mu}=\frac{1}{2}\left(D_{\mathrm{m}} \varepsilon^{\mu}+\omega_{\mathrm{m}}{ }^{n l}\left(\gamma_{n l} \varepsilon\right)^{\mu}-E^{\mu}{ }_{\underline{\nu}} \partial_{\mathrm{m}} \varepsilon^{\underline{\nu}}+F_{\mathrm{NS}}{ }^{\mu \nu}\left(\gamma_{\mathrm{m}} \varepsilon\right)_{\nu}+F_{\mathrm{RR}}{ }^{\mu \nu^{\prime}}\left(\gamma_{\mathrm{m}} \varepsilon\right)_{\nu^{\prime}}\right) \\
& \delta_{\varepsilon} \psi_{\mathrm{m}}{ }^{\mu^{\prime}}=\frac{1}{2}\left(D_{\mathrm{m}} \varepsilon^{\mu^{\prime}}-\omega_{\mathrm{m}}{ }^{\prime} l^{\prime}\left(\gamma_{n^{\prime} l^{\prime} \varepsilon}\right)^{\mu^{\prime}}-E^{\mu^{\prime}} \partial_{\mathrm{m}} \varepsilon^{\underline{\nu}}+F_{\mathrm{NS}} \mu^{\mu^{\prime} \nu^{\prime}}\left(\gamma_{\mathrm{m}} \varepsilon\right)_{\nu^{\prime}}+F_{\mathrm{RR}}{ }^{\mu^{\prime} \nu}\left(\gamma_{\mathrm{m}} \varepsilon\right)_{\nu}\right) \\
& \delta_{\varepsilon} E_{\mathrm{F}}=E_{\mathrm{F}}\left(\mu \varepsilon^{\left.\nu^{\prime}\right)}\right. \\
& \delta_{\varepsilon} E_{\mathrm{F}}{ }^{\mu}=\delta_{\varepsilon} E^{\nu \nu^{\prime} ; \mu}=C_{\mathrm{RR}} \underline{m}^{\nu \nu^{\prime}} \partial^{\underline{m}} \varepsilon^{\mu}+E_{\mathrm{F}}{ }^{m n}\left(\gamma_{m n} \varepsilon\right)^{\mu}-E^{\mu(\nu} \varepsilon^{\left.\nu^{\prime}\right)}
\end{aligned}
$$

It is noted that spinor indices are denoted by ${ }^{\mu, \nu, \cdots}$, since our flat Lorentz indices are denoted by $S_{m n}$ and $S_{m^{\prime} n^{\prime}}$. But later ${ }^{\alpha, \beta, \cdots}$ is used after gauge fixing $S_{m n}=S_{a b}$ and $S_{m^{\prime} n^{\prime}}=S_{a^{\prime} b^{\prime}}$. From the supersymmetry transformation of $C_{\mathrm{RRm}}{ }^{\mu \nu^{\prime}}, E_{\mathrm{F} \Omega}$ turns out to include dilatino $\lambda$. It is also derived that $E_{\mathrm{FF}}$ include dilaton $\phi$ from its supersymmetry transformation rule. Unlike the case of type II superspace without $\Upsilon_{\mu \nu^{\prime}}$, the dilaton and the dilatino are included in vielbein field without introducing $\hat{T}$ constraint which is obtained from the integral measure factor.

From the supersymmetry transformation rule of the gravitinos $E_{\Omega \Omega}$ are identified with NS-NS and R-R gauge field strengths, $E_{\Omega \Omega}=E_{\mathrm{NS}}{ }^{\mu \nu}$ and $E_{\Omega \Omega^{\prime}}=E_{\mathrm{RR}}{ }^{\mu \nu^{\prime}}$ as same as before. In addition to this the R-R gauge field strengths are included in $E_{\mathrm{F} \Sigma}=F_{\mathrm{RR}}{ }^{\alpha \beta^{\prime} c d}$ from the supersymmetry transformation of the dilatino in $E_{\mathrm{F} \Omega}$. The R-R field strength $F_{\mathrm{RR}}$ appears

\begin{tabular}{|c|c|}
\hline type II string field & fields (dim.) \\
\hline gravitational metric & $e_{(\underline{a m})}=E_{(\underline{a m})}, e_{\underline{\alpha}} \underline{\mu}=E_{\underline{\alpha} \Omega}(0)$ \\
\hline$B$ fields & $B_{\underline{\alpha \mu}}=E_{\underline{\alpha \mu}}(-1), B_{\underline{a \mu}}=E_{\underline{a \mu}}\left(-\frac{1}{2}\right), B_{\underline{a m}}=E_{[\underline{a m}]}(0)$ \\
\hline$C$ fields & $C_{\underline{\alpha}^{\mu}}^{\mu \nu^{\prime}}=E_{\underline{\alpha} \boldsymbol{F}}\left(-\frac{1}{2}\right), C_{\underline{a}^{\mathrm{RR}}}^{\mu \nu^{\prime}}=E_{\underline{a}}(0)$ \\
\hline dilaton & $\varphi=E_{\mathrm{FF}}(0)$ \\
\hline dilatino & $\lambda_{\underline{\mu}}=E_{\mathrm{F} \Omega}, E_{\underline{a} \Omega}\left(\frac{1}{2}\right)$ \\
\hline gravitino & $\psi_{\underline{a}} \underline{\mu}=E_{\underline{a} \Omega}\left(\frac{1}{2}\right)$ \\
\hline superconnections & $\omega_{\underline{\alpha}} \underline{m n}=E_{\underline{\alpha} \Sigma}\left(\frac{1}{2}\right), \omega_{\underline{\alpha}} \underline{m n}=E_{\underline{a} \Sigma}(1), \omega^{\underline{\alpha m n}}=E_{\Omega \Sigma}\left(\frac{3}{2}\right)$ \\
\hline NS-NS field strength & $F_{\mathrm{NS}}{ }^{[\mu \nu]}, F_{\mathrm{NS}}\left[\mu^{\prime} \nu^{\prime}\right]=E_{[\Omega \Omega]}, E_{[\underline{a} \Sigma]}(1)$ \\
\hline $\mathrm{R}-\mathrm{R}$ field strength & $F_{\mathrm{RR}}{ }^{\mu \nu^{\prime}}=E_{\Omega \Omega^{\prime}}, E_{\mathrm{F} \Sigma}(1)$ \\
\hline covariance compensator & $r \frac{a b m n}{=E_{\Sigma \Sigma}}(2)$ \\
\hline
\end{tabular}
in $\left\{\triangleright_{\alpha}, \triangleright_{\beta^{\prime}}\right\}$, so $T_{\alpha \beta^{\prime} \Sigma}$ and $T_{a \alpha}{ }^{\beta^{\prime}}$ are related by the Bianchi identity. These torsions were not zero without central extension, but they are absorbed by vielbein as $T_{\alpha \beta^{\prime} \Sigma}=f_{\alpha \beta \digamma} E_{\Upsilon \Sigma}$ and $T_{a \alpha}{ }^{\beta^{\prime}}=f_{a \alpha \gamma} E^{\gamma \beta^{\prime}}$.

As a result vielbein fields are identified to supergravity multiplets from Bianchi identities, torsion constraints and gauge transformations as follows: 


\subsection{Linearized solution}

The vielbein in a weak field expansion with lower indices, $E_{\underline{A M}}=E_{\underline{A}} \underline{\underline{N}} \eta_{\underline{N M}}$, are written as

$$
\left.E_{\underline{A M}}=\eta_{\underline{A M}}+H_{\underline{A}}^{\underline{B}} \eta_{\underline{B M}} \Rightarrow H_{\underline{A B}}=H_{\underline{A}} \underline{C}_{\underline{C B}}=\frac{1}{2} H_{[\underline{A B}}\right) .
$$

Coordinates corresponding to Lorentz symmetries and the R-R central charge are auxiliary coordinates, so it is possible to choose $E_{\underline{S M}}=\eta_{\underline{S M}}$ and $E_{\Upsilon \underline{M}}=\eta_{\Upsilon \underline{M}}$. The vielbein fields given by (4.13) in linearized level are also denoted as

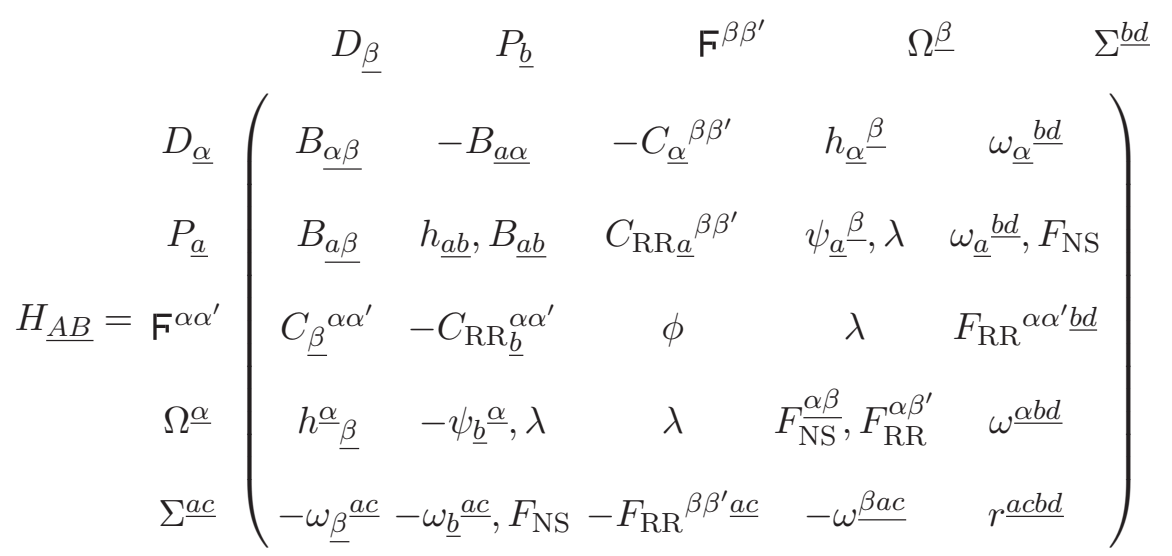

Torsions in linearized level are given by

$$
T_{\underline{A B C}}=\frac{1}{2}\left(D_{[\underline{A}} H_{\underline{B C]})}-H_{[\underline{A}} \underline{\underline{D}} f_{\underline{B C] D}}\right)
$$

where $D_{\underline{A}}$ is a flat space covariant derivative. The generalized gauge transformations are

$$
\delta_{\Lambda} H_{\underline{A B}}=D_{[\underline{A}} \Lambda_{\underline{B})}-\Lambda^{\underline{C}} f_{\underline{C A B}} .
$$

Let us solve torsion constraints given by (4.7) in the linearized level. Although there are new torsions appeared by the central extension with indices $\Upsilon$ and $\mathbf{F}$, many torsion constraints whose indices do not include $\Upsilon$ and $F$ are the same as the previous paper [18] as;

$$
\begin{aligned}
T_{\underline{A B C}} & =\frac{1}{2}\left(D_{[\underline{A}} H_{\underline{B C}]}+H_{[\underline{A}} \underline{D} f_{\underline{D} \mid \underline{B C}]}+H_{[\underline{A} \mid \Upsilon} f_{\mathrm{F} \mid \underline{B C}]}+H_{[\underline{A \mid \mathrm{F}}} f_{\Upsilon \mid \underline{B C}]}\right) \\
& =\frac{1}{2}\left(D_{[\underline{A}} H_{\underline{B C}]}+H_{[\underline{A}} \underline{D} f_{\underline{D} \mid \underline{B C}]}\right) \quad, \underline{A \neq \Upsilon, \mathrm{F}}
\end{aligned}
$$

because of $H_{\Upsilon \underline{A}}=f_{\Upsilon \underline{A B}}=0$. Torsions with two $\Upsilon$ indices are zero because of the same reason

$$
T_{\underline{A} \Upsilon \Upsilon}=\frac{1}{2}\left(D_{[\underline{A}} H_{\Upsilon \Upsilon]}+H_{\left[\underline{A}^{\underline{D}}\right.} f_{\underline{D} \mid \underline{\Upsilon \Upsilon}]}\right)=0 .
$$

Therefore nontrivial new torsions are

$$
T_{\Upsilon \underline{A B}}, T_{\Upsilon \mathrm{F} \underline{A}}, T_{\Upsilon \mathrm{FF}}, T_{\mathrm{F} \underline{A B}}, T_{\mathrm{FF} \underline{A}}, T_{\mathrm{FFF}} \text { for } \underline{A}, \underline{B} \neq \Upsilon \mathrm{F}
$$


New torsion constraints involving $\Upsilon$ and $\mathrm{F}$ indices are solved in linearized level:

\begin{tabular}{|c|c|c|}
\hline \multicolumn{3}{|c|}{ dim torsion constraints $\Rightarrow$ field } \\
\hline \multirow[t]{3}{*}{0} & $T_{\alpha \underline{\beta} \Upsilon}=0$ & $D_{\Upsilon} B_{\alpha \underline{\beta}}=0$ \\
\hline & $T_{\alpha \beta \digamma}=0$ & $C^{\mathrm{RR}}{ }_{a}^{\gamma \gamma^{\prime}}=-D_{(\alpha} C_{\beta)} \gamma \gamma^{\prime}\left(\gamma_{a}\right)^{\alpha \beta}-h_{\alpha}{ }^{\gamma^{\prime}}\left(\gamma_{a}\right)^{\alpha \gamma}$ \\
\hline & $T_{\alpha \beta^{\prime} \mathrm{F}}=f_{\alpha \beta^{\prime} \mathrm{F}}$ & $h_{\alpha}^{\gamma} f_{\beta^{\prime} \gamma \mathrm{F}}-h_{\beta^{\prime}} \gamma^{\prime} f_{\alpha \gamma^{\prime} \mathrm{F}}=D_{(\alpha} C_{\left.\beta^{\prime}\right) \mathrm{F}}$ \\
\hline \multirow[t]{5}{*}{$\frac{1}{2}$} & $T_{\alpha \underline{b} \Upsilon}=0$ & $D_{\Upsilon} B_{\alpha \underline{b}}=0$ \\
\hline & $T_{\alpha \Upsilon F}=0$ & $\omega_{\alpha \Sigma}=-D_{\Upsilon} C_{\alpha F}$ \\
\hline & $T_{\alpha a F}=0$ & $\psi_{a}^{\beta^{\prime}} f_{\alpha \beta^{\prime} F}+H_{F}{ }^{\beta}\left(\gamma_{a}\right)_{\alpha \beta}=D_{\alpha} C_{a \text { F }}^{\mathrm{RR}}-D_{a} C_{\alpha \mathrm{F}}$ \\
\hline & $T_{\alpha a^{\prime} \digamma}=0$ & $\psi_{a^{\prime}}^{\beta^{\prime}} f_{\alpha \beta^{\prime} \boldsymbol{F}}=-D_{\alpha} C_{a^{\prime} \boldsymbol{F}}^{\mathrm{RR}}+D_{a^{\prime}} C_{\alpha \boldsymbol{F}}$ \\
\hline & $T_{\alpha \digamma \digamma}=0$ & 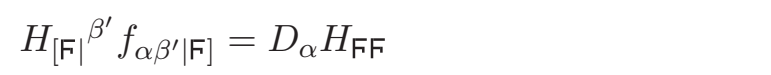 \\
\hline \multirow[t]{9}{*}{1} & $T_{\alpha} \Upsilon \underline{\beta}=0$ & $D_{\Upsilon} h^{\beta_{\alpha}}=0$ \\
\hline & $T_{a \underline{b} \Upsilon} \Upsilon=0$ & $D_{\Upsilon} h_{a \underline{b}}=0$ \\
\hline & $T_{a \Upsilon F}=0$ & $\omega_{a \Sigma}=-D_{\Upsilon} C_{a \digamma}^{\mathrm{RR}}$ \\
\hline & $T_{\Upsilon F F}=0$ & $F_{\mathrm{RRF} \Sigma}=D_{\Upsilon} H_{\mathrm{FF}}$ \\
\hline & $T_{\alpha \mathrm{F}^{\beta}}{ }^{\beta} 0$ & $F_{\mathrm{RRF}}{ }^{a b}\left(\gamma_{a b}\right)^{\beta}{ }_{\alpha}-F_{\mathrm{RR}}^{\beta \beta^{\prime}} f_{\alpha \beta^{\prime}}=-D_{\alpha} H_{\mathrm{F}}{ }^{\beta}$ \\
\hline & $T_{\alpha \mathrm{F}^{\beta^{\prime}}}=0$ & $F_{\mathrm{NS}}^{\alpha \beta^{\prime}} f_{\alpha \beta^{\prime} \mathrm{F}}=-D_{\alpha} H_{\mathrm{F}}^{\beta^{\prime}}$ \\
\hline & $T_{a b \digamma}=0$ & $F_{\mathrm{RR} F a b}=-D_{[a} C_{b] \overline{\mathrm{RR}}}^{\mathrm{RR}}$ \\
\hline & $T_{a b^{\prime} \mathrm{F}}=0$ & $D_{[a} C_{\left.b^{\prime}\right] \mathrm{F}}^{\mathrm{RR}}=0$ \\
\hline & $T_{a \mathrm{FF}}=0$ & $D_{a} H_{\mathrm{FF}}=0$ \\
\hline \multirow[t]{4}{*}{$3 / 2$} & $T_{a \Upsilon^{\beta^{\prime}}}=0$ & $D_{\Upsilon} \psi_{a}^{\beta^{\prime}}=0$ \\
\hline & $T_{\Upsilon F}{ }^{\beta}=0$ & $\omega^{\beta} f_{S \Upsilon F}=D_{\Upsilon} H_{\mathrm{F}}{ }^{\beta}$ \\
\hline & $T_{a \mathrm{~F}^{\beta}}=0$ & $D_{a} H_{F^{\beta}}^{\beta}=0$ \\
\hline & $T_{\alpha \mathrm{F \Sigma}}=0$ & $\omega^{\beta^{\prime} a b}=D_{\alpha} F_{\mathrm{RR}}^{\alpha \beta^{\prime} a b}$ \\
\hline \multirow[t]{2}{*}{2} & $T_{\Upsilon}^{\alpha \beta}=0$ & $D_{\Upsilon} F_{\mathrm{NS}}{ }^{\alpha \beta}=0$ \\
\hline & $T_{a \Upsilon \Sigma}=0$ & $D_{\Upsilon} \omega_{a}^{b c}=0$ \\
\hline
\end{tabular}

where we assumed $D_{\Sigma} E=D_{\Omega} E=D_{\mathrm{F}} E=0$ as a simple expression although it is not necessary. 


\section{Green-Schwarz superstring action}

In this section we write down actions for strings in the manifestly T-duality formalism. The Wess-Zumino terms are written by bilinear combination of left-invariant local currents. Equations of motion are chirally separated.

\subsection{Action}

For a graded Lie algebra $G_{\underline{M}}$

$$
\left[G_{\underline{M}}, G_{\underline{N}}\right\}=i f_{\underline{M N}} \underline{\underline{K}} G_{\underline{K}},
$$

the left-invariant current $J$ is written as

$$
\begin{aligned}
g^{-1}(d g) & =J, & d J & =-J \wedge J \\
J & =i J^{\underline{M}} G_{\underline{M}}, & d J^{\underline{M}} & =-\frac{1}{2} J^{\underline{K}} \wedge J^{\underline{N}} f_{\underline{N K}} \underline{M} .
\end{aligned}
$$

It is related to the covariant derivative as

$$
g^{-1}(d g)=i d Z^{\underline{M}} E_{\underline{M}}^{\underline{A}} G_{\underline{A}}, \quad \nabla_{\underline{A}}=\frac{1}{i} E_{\underline{A}}^{\underline{M}} \partial_{\underline{M}} .
$$

It is convenient to classify the left-invariant currents by the canonical dimensions of generators denoted by $s$, where they satisfy following Maurer-Cartan (MC) equations for left-invariant currents

$$
J=\sum_{s=0}^{2} J_{[s]}, \quad d J_{[s]}=-\sum_{t=0}^{s} J_{[s-t]} \wedge J_{[t]} .
$$

Right-invariant currents are decomposed as follows and they satisfy the following MC equations for $j$

$$
\begin{aligned}
(d g) g^{-1} & =j, \quad d j=j \wedge j \\
j_{[s]} & \equiv g J_{[s]} g^{-1}, \quad j=\sum_{s=0}^{2} j_{[s]}, \quad d j_{[s]}=j \wedge j_{[s]}+j_{[s]} \wedge j-\sum_{t=0}^{s} j_{[s-t]} \wedge j_{[t]} .
\end{aligned}
$$

It is noted that $j_{[s]}$ does not have a definite dimension $s$, but it may contain several generators with different dimension $s$.

We introduce three kinds of traces;

$$
\begin{array}{ll}
\operatorname{trace} & \text { rank } \\
\operatorname{tr}\left(G_{\underline{M}} G_{\underline{N}}\right)=\eta_{\underline{M N}} & \text { nondegenerate, } \eta_{\underline{M}} \\
\hat{\operatorname{tr}}\left(G_{\underline{M}} G_{\underline{N}}\right) \equiv \hat{\eta}_{\underline{M N}} & \text { not always nondege } \\
\left.\operatorname{tr}_{B}\left(G_{\underline{M}} G_{\underline{N}}\right) \equiv \eta_{\underline{M N}}\right|_{\underline{M} \in \tilde{I}, \underline{N} \in I} \text { almost half }- \text { rank }
\end{array}
$$

reference eq. 
Three kinds of "traces" are non-vanishing only if $\operatorname{dim}\left(G_{\underline{M}}\right)+\operatorname{dim}\left(G_{\underline{N}}\right)=2$. Indices $I$ and $\tilde{I}$ are classified in (2.1). Cyclicity holds for $\operatorname{tr}(g h)=\operatorname{tr}(h g)$. The structure constant with lower indices is totally graded-antisymmetric

$$
\operatorname{tr}\left(\left[G_{\underline{M}}, G_{\underline{N}}\right\} G_{\underline{K}}\right)=i f_{\underline{M N K}},
$$

which are nonvanishing only if $\operatorname{dim}\left(G_{\underline{M}}\right)+\operatorname{dim}\left(G_{\underline{N}}\right)+\operatorname{dim}\left(G_{\underline{K}}\right)=2$.

We propose the following Green-Schwarz action in manifestly T-duality formalism,

$$
\begin{aligned}
I & =\int d \tau d \sigma\left[\mathcal{L}_{0}+\mathcal{L}_{\mathrm{WZ}}\right] \\
\mathcal{L}_{0} & =\frac{1}{2} J_{[1]}^{\underline{M}} \wedge * J_{[1]}^{\underline{N}} \hat{\eta}_{\underline{M N}}=-\frac{1}{2} \hat{\operatorname{tr}} J_{[1]} \wedge * J_{[1]} \\
\mathcal{L}_{\mathrm{WZ}} & =\frac{\mathcal{K}}{2} J^{\underline{M}} \wedge J^{\underline{N}} B_{\underline{M N}}=\mathcal{K} \sum_{s=0}^{1} J_{[2-s]}^{\tilde{J}} \wedge J_{[s]}^{I} \eta_{\tilde{I} J}=-\mathcal{K} \sum_{s=0}^{1} \operatorname{tr}_{B} J_{[2-s]} \wedge J_{[s]} .
\end{aligned}
$$

with $\mathcal{K}= \pm 1$. The $B_{\underline{M N}}$ fields in flat space are graded antisymmetric constants as

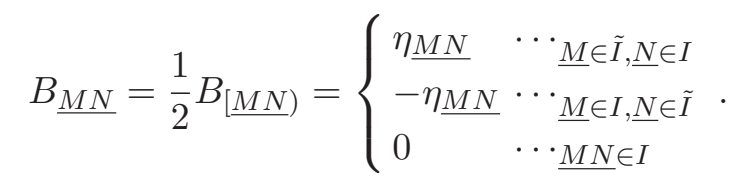

Even for a bosonic string it is useful to include the Wess-Zumino term with respect to fluxes corresponding to structure constants of the nondegenerate algebra. Currents included in the Wess-Zumino term do not appear in the kinetic term $\mathcal{L}_{0}$, so they are auxiliary degrees of freedom. Dimensional reduction constraints are imposed to remove these auxiliary coordinates.

Let us evaluate the exterior derivative of the Wess-Zumino term ;

$$
\begin{aligned}
d \mathcal{L}_{\mathrm{WZ}} & =-d\left(\mathcal{K} \sum_{s=0}^{1} \operatorname{tr}_{B} J_{[2-s]} \wedge J_{[s]}\right) \\
& =-\mathcal{K} \sum_{s=0}^{1} \operatorname{tr}_{B}\left(d J_{[2-s]} \wedge J_{[s]}-J_{[2-s]} \wedge d J_{[s]}\right) \\
& =-\mathcal{K} \sum_{s=0}^{1} \operatorname{tr}_{B}\left(-\sum_{t=0}^{2-s} J_{[2-s-t]} \wedge J_{[t]} \wedge J_{[s]}+\sum_{t=0}^{s} J_{[2-s]} \wedge J_{[s-t]} \wedge J_{[t]}\right) \\
& =\mathcal{K}\left[\frac{1}{3} \operatorname{tr} J \wedge J \wedge J+\operatorname{tr}_{B}\left(\sum_{t=\frac{1}{2}}^{1} J_{[1]} \wedge J_{[1-t]} \wedge J_{[t]}\right)\right]
\end{aligned}
$$

The Wess-Zumino term is Chevalley-Eilenberg cohomology trivial in the centrally extended algebra, while non-trivial in usual case. The usual Green-Schwarz superstring action has only second term in the right hand side of (5.7), where the Wess-Zumino term cannot be written down in bilinear form of the currents locally in 2-dimensional worldsheet. The first term in (5.7) gives all fluxes corresponding to structure constants of the global symmetry 
algebra. It turns out that this term makes (5.7) exact three form and the Wess-Zumino term is obtained in the bilinear of currents as in (5.5).

Under an arbitrary variation the left-invariant current becomes

$$
\delta J=\delta\left(g^{-1} d g\right)=g^{-1}(d \Delta) g, \quad \Delta=\delta g g^{-1} .
$$

The variation of the first term of (5.7) is total derivative written in terms of one form currents as

$$
\delta \frac{1}{3} \operatorname{tr} J \wedge J \wedge J=-d(\operatorname{tr}(\Delta d j)),
$$

which gives a contribution of current divergence to an equation of motion.

We take variation of the local form of the Wess-Zumino term in (5.5) directly:

$$
\begin{aligned}
\delta \mathcal{L}_{\mathrm{WZ}} & =\mathcal{K} \operatorname{tr}_{B} \Delta \sum_{s=0}^{1} d\left(j_{[2-s]}-j_{[s]}\right) \\
& =\mathcal{K}\left[\operatorname{tr} \Delta\left(-d j+\sum_{0<t<2} \sum_{0<r<t} j_{[t-r]} \wedge j_{[r]}\right)+\operatorname{tr}_{B} \Delta \sum_{s>0}^{1} d\left(j_{[2-s]}-j_{[s]}\right)\right]
\end{aligned}
$$

The variation of the kinetic term becomes

$$
\delta \mathcal{L}_{0}=\hat{\operatorname{tr}}\left[\Delta d * j_{[1]}\right] .
$$

\section{$5.2 \quad$ T-dual bosonic string}

One form currents of a bosonic string are denoted by

$$
\begin{aligned}
J_{[0]} & =\left\{S, S^{\prime}\right\}, \quad J_{[1]}=\left\{P, P^{\prime}\right\}, \quad J_{[2]}=\left\{\Sigma, \Sigma^{\prime}\right\} \\
j_{[0]} & =\left\{s, s^{\prime}\right\}, \quad j_{[1]}=\left\{p, p^{\prime}\right\}, \quad j_{[2]}=\left\{\sigma, \sigma^{\prime}\right\} \\
j & =s+p+\sigma, \quad j^{\prime}=s^{\prime}+p^{\prime}+\sigma^{\prime}
\end{aligned}
$$

For cases where two sets of algebras are decoupled, $J$ and $J^{\prime}, j$ and $j^{\prime}$ are functions only on $Z^{M}$ and $Z^{M^{\prime}}$ because of $g=g\left(Z^{M}\right) g\left(Z^{M^{\prime}}\right)=g\left(Z^{M^{\prime}}\right) g\left(Z^{M}\right)$. The left-invariant currents satisfy the following $\mathrm{MC}$ equations

$$
\begin{aligned}
& g^{-1} d g=J+J^{\prime} \\
& J=S+P+\Sigma, J^{\prime}=S^{\prime}+P^{\prime}+\Sigma^{\prime} \\
& \left\{\begin{array}{l}
d S=-S \wedge S \\
d P=-P \wedge S-S \wedge P \\
d \Sigma=-\Sigma \wedge S-S \wedge \Sigma-P \wedge P \\
0=P \wedge \Sigma+\Sigma \wedge P=\Sigma \wedge \Sigma
\end{array},\left\{\begin{array}{l}
d S^{\prime}=-S^{\prime} \wedge S^{\prime} \\
d P^{\prime}=-P^{\prime} \wedge S^{\prime}-S^{\prime} \wedge P^{\prime} \\
d \Sigma^{\prime}=-\Sigma^{\prime} \wedge S^{\prime}-S^{\prime} \wedge \Sigma^{\prime}-P^{\prime} \wedge P^{\prime} \\
0=P^{\prime} \wedge \Sigma^{\prime}+\Sigma^{\prime} \wedge P^{\prime}=\Sigma^{\prime} \wedge \Sigma^{\prime}
\end{array}\right.\right.
\end{aligned}
$$

Components of the right invariant current satisfy the following MC equations

$$
(d g) g^{-1}=j+j^{\prime}
$$




$$
\begin{aligned}
& j=s+p+\sigma, j^{\prime}=s^{\prime}+p^{\prime}+\sigma^{\prime} \\
& \left\{\begin{array}{l}
d s=j \wedge j-p \wedge p \\
d p=2 p \wedge p \\
d \sigma=-p \wedge p \\
0=p \wedge \sigma+\sigma \wedge p=\sigma \wedge \sigma
\end{array},\left\{\begin{array}{l}
d s^{\prime}=j^{\prime} \wedge j^{\prime}-p^{\prime} \wedge p^{\prime} \\
d p^{\prime}=2 p^{\prime} \wedge p^{\prime} \\
d \sigma^{\prime}=-p^{\prime} \wedge p^{\prime} \\
0=p^{\prime} \wedge \sigma^{\prime}+\sigma^{\prime} \wedge p^{\prime}=\sigma^{\prime} \wedge \sigma^{\prime}
\end{array}\right.\right.
\end{aligned}
$$

An action for a bosonic string in the manifestly T-duality formalism is given by

$$
\begin{aligned}
\mathcal{L}_{0} & =-\frac{1}{2} \hat{\operatorname{tr}}\left[P \wedge * P+P^{\prime} \wedge * P^{\prime}\right]=-\frac{1}{2} \operatorname{Tr}\left[P \wedge * P+P^{\prime} \wedge * P^{\prime}\right] \\
\mathcal{L}_{\mathrm{WZ}} & =-\mathcal{K} \operatorname{tr}_{B}\left[\Sigma \wedge S+\Sigma^{\prime} \wedge S^{\prime}\right]=-\mathcal{K} \operatorname{Tr}\left[\Sigma \wedge S-\Sigma^{\prime} \wedge S^{\prime}\right]
\end{aligned}
$$

with $\operatorname{Tr}\left(G_{M} G_{N}\right)=\eta_{M N}$ and $\operatorname{Tr}\left(G_{M^{\prime}} G_{N^{\prime}}\right)=\left|\eta_{M^{\prime} N^{\prime}}\right|$. From the MC equation (5.14)

$$
d\left(j_{[2]}-j_{[0]}\right)=d\left(\sigma-s+\sigma^{\prime}-s^{\prime}\right)=-d\left(j+j^{\prime}\right),
$$

the variation of the action is given by

$$
\begin{aligned}
\delta \mathcal{L}_{0} & =\hat{\operatorname{tr}}\left[\Delta d *\left(p+p^{\prime}\right)\right]=\operatorname{Tr}\left[\Delta d *\left(p+p^{\prime}\right)\right] \\
\delta \mathcal{L}_{\mathrm{WZ}} & =-\mathcal{K} \operatorname{tr}\left[\Delta d\left(j+j^{\prime}\right)\right]=-\mathcal{K} \operatorname{Tr}\left[\Delta d\left(j-j^{\prime}\right)\right]
\end{aligned}
$$

with $* *=1$. As a result the equation of motion is given by

$$
d *(p-\mathcal{K} *(p+\sigma+s))=0, d *\left(p^{\prime}+\mathcal{K} *\left(p^{\prime}+\sigma^{\prime}+s^{\prime}\right)\right)=0
$$

or equivalently

$$
\partial_{i}\left(p^{i}-\mathcal{K} \epsilon^{i j}(p+\sigma+s)_{j}\right)=0, \partial_{i}\left(p^{i}+\mathcal{K} \epsilon^{i j}\left(p^{\prime}+\sigma^{\prime}+s^{\prime}\right)_{j}\right)=0, \quad i, j=\tau, \sigma .
$$

Since left and right sets are decoupled, the left and the right coordinates satisfy equations of motion independently. The nondegenerate metric $\eta_{M N}$ has opposite signatures for left and right coordinates, so the coefficients of the Wess-Zumino term have opposite signs for left and right sectors. The equations of motions for left and right sectors become different chirality with $\partial_{ \pm}=\partial_{\sigma} \pm \partial_{\tau}$ and $j_{ \pm}=j_{\sigma} \pm j_{\tau}$ as

$$
\begin{gathered}
\mathcal{K}=-1 \Rightarrow\left\{\begin{array}{l}
\partial_{+}\left(p_{-}+\frac{1}{2}\left(s_{-}+\sigma_{-}\right)\right)-\frac{1}{2} \partial_{-}\left(s_{+}+\sigma_{+}\right)=0 \\
\partial_{-}\left(p_{+}^{\prime}+\frac{1}{2}\left(s_{+}^{\prime}+\sigma_{+}^{\prime}\right)\right)-\frac{1}{2} \partial_{+}\left(s_{-}^{\prime}+\sigma_{-}^{\prime}\right)=0
\end{array}\right. \\
\text { or } \\
\mathcal{K}=1 \Rightarrow\left\{\begin{array}{l}
\partial_{-}\left(p_{+}+\frac{1}{2}\left(s_{+}+\sigma_{+}\right)\right)-\frac{1}{2} \partial_{+}\left(s_{-}+\sigma_{-}\right)=0 \\
\partial_{+}\left(p_{-}^{\prime}+\frac{1}{2}\left(s_{-}^{\prime}+\sigma_{-}^{\prime}\right)\right)-\frac{1}{2} \partial_{-}\left(s_{+}^{\prime}+\sigma_{+}^{\prime}\right)=0
\end{array}\right.
\end{gathered}
$$

Since coordinates of Lorentz symmetry $S_{m n}, S_{m^{\prime} n^{\prime}}$ and their nondegenerate partners $\Sigma^{m n}$, $\Sigma^{m^{\prime} n^{\prime}}$ do not have kinetic terms, their coordinates are auxiliary variables. For a coset 
space Poincaré/Lorentz, $S_{m n}=S_{m^{\prime} n^{\prime}}=0$ are coset constraints and $\tilde{\Sigma}^{m n}$ and $\tilde{\Sigma}^{m^{\prime} n^{\prime}}$ are also constrained as gauge fields [42-44]. From the algebra (2.6) both $S_{m n}=0$ and $\Sigma_{m n}=0$ are not first class constraints, but $S_{m n}=0$ and the symmetry generator $\tilde{\Sigma}^{\mu \nu^{\prime}}=0$ are first class constraints where symmetry generators commute with covariant derivatives. Then currents $J_{[0]}=S, S^{\prime}$ and $j_{[2]}=\sigma, \sigma^{\prime}$ are gauged away as gauge fixing conditions. Details are discussed in another paper [48]. Then left/right currents $p_{i}$ and $p_{i}^{\prime}$ are essentially chiral currents, e.g. $p_{+}=\partial_{+} x$ and $p_{-}^{\prime}=\partial_{-} x^{\prime}$ in flat space for $\mathcal{K}=1$. Equation of motion for bosonic coordinates are $\partial_{-} p_{+}=\partial_{-}\left(\partial_{+} x\right)=0$ and $\partial_{+} p_{-}=\partial_{+}\left(\partial_{-} x^{\prime}\right)=0$. The dimensional reduction condition reduces double coordinates into the usual one, for example $p-p^{\prime}=0$ and $X=x+x^{\prime}$, then equations of motion reduce into the usual one, $\partial_{+} \partial_{-} X=0$.

\subsection{Type II superstring}

For a type II superstring one form currents of the nondegenerate super-Poincaré algebra are denoted by

$$
\begin{aligned}
& J_{[0]}=\left\{S, S^{\prime}\right\}, \quad J_{\left[\frac{1}{2}\right]}=\left\{D, D^{\prime}\right\}, \quad J_{[1]}=\left\{P, P^{\prime}\right\}, \quad J_{\left[\frac{3}{2}\right]}=\left\{\Omega, \Omega^{\prime}\right\}, \quad J_{[2]}=\left\{\Sigma, \Sigma^{\prime}\right\} \\
& j_{[0]}=\left\{s, s^{\prime}\right\}, \quad j_{\left[\frac{1}{2}\right]}=\left\{\mathrm{d}, \mathrm{d}^{\prime}\right\}, \quad j_{[1]}=\left\{p, p^{\prime}\right\}, \quad j_{\left[\frac{3}{2}\right]}=\left\{\omega, \omega^{\prime}\right\}, \quad j_{[2]}=\left\{\sigma, \sigma^{\prime}\right\} .
\end{aligned}
$$

The left-invariant currents and the MC equations are

$$
\begin{aligned}
g^{-1} d g & =J+J^{\prime} \\
J & =S+D+P+\Omega+\Sigma, J^{\prime}=S^{\prime}+D^{\prime}+P^{\prime}+\Omega^{\prime}+\Sigma^{\prime} \\
d S & =-S \wedge S \\
d D & =-S \wedge D-D \wedge S \\
d P & =-S \wedge P-P \wedge S-D \wedge D \\
d \Omega & =-S \wedge \Omega-\Omega \wedge S-D \wedge P-P \wedge D \\
d \Sigma & =-S \wedge \Sigma-\Sigma \wedge S-D \wedge \Omega-\Omega \wedge D-P \wedge P \\
0 & =\Sigma \wedge \Sigma=\Sigma \wedge \Omega+\Omega \wedge \Sigma=\Sigma \wedge P+P \wedge \Sigma \\
& =\Sigma \wedge D+D \wedge \Sigma=\Omega \wedge \Omega=\Omega \wedge P+P \wedge \Omega .
\end{aligned}
$$

The $\mathrm{MC}$ equations for primed left-invariant currents are the same as above. Decomposed right-invariant currents and the $\mathrm{MC}$ equations are

$$
\begin{aligned}
(d g) g^{-1} & =j+j^{\prime} \\
j & =s+\mathrm{d}+p+\omega+\sigma, j^{\prime}=s^{\prime}+\mathrm{d}^{\prime}+p^{\prime}+\omega^{\prime}+\sigma^{\prime} \\
d s & =j \wedge j-p \wedge p-\mathrm{d} \wedge \mathrm{d}-\mathrm{d} \wedge(p+\omega)-(p+\omega) \wedge \mathrm{d} \\
d \mathrm{~d} & =2 \mathrm{~d} \wedge \mathrm{d}+\mathrm{d} \wedge(p+\omega)+(p+\omega) \wedge \mathrm{d} \\
d p & =-\mathrm{d} \wedge \mathrm{d}+2 p \wedge p+\mathrm{d} \wedge p+p \wedge \mathrm{d} \\
d \omega & =\mathrm{d} \wedge(-p+\omega)+(-p+\omega) \wedge \mathrm{d} \\
d \sigma & =-p \wedge p-\mathrm{d} \wedge \omega-\omega \wedge \mathrm{d} \\
0 & =\sigma \wedge \sigma=\sigma \wedge \omega+\omega \wedge \sigma=\sigma \wedge p+p \wedge \sigma
\end{aligned}
$$




$$
=\sigma \wedge \mathrm{d}+\mathrm{d} \wedge \sigma=\omega \wedge \omega=\omega \wedge p+p \wedge \omega .
$$

The MC equations for primed ones are the same as the above with replacing unprimed indices with primed indices.

An action for a superstring in the manifestly T-duality formalism is given by

$$
\begin{aligned}
\mathcal{L}_{0} & =-\frac{1}{2} \hat{\operatorname{tr}}\left[P \wedge * P+P^{\prime} \wedge * P^{\prime}\right]=-\frac{1}{2} \operatorname{Tr}\left[P \wedge * P+P^{\prime} \wedge * P^{\prime}\right] \\
\mathcal{L}_{\mathrm{WZ}} & =-\mathcal{K} \operatorname{tr}_{B}\left[\Sigma \wedge S+\Omega \wedge D+\Sigma^{\prime} \wedge S^{\prime}+\Omega^{\prime} \wedge D^{\prime}\right] \\
& =-\mathcal{K} \operatorname{Tr}\left[\Sigma \wedge S+\Omega \wedge D-\Sigma^{\prime} \wedge S^{\prime}-\Omega^{\prime} \wedge D^{\prime}\right] .
\end{aligned}
$$

From the MC equations in (5.21)

$$
\begin{aligned}
d(\sigma-s+\omega-\mathrm{d}) & =-j \wedge j-\mathrm{d} \wedge \mathrm{d}-p \wedge \mathrm{d}-\mathrm{d} \wedge p \\
& =-d\left(j+\frac{1}{2}(-\omega+\mathrm{d})\right) \\
& =-d\left(p+\frac{3}{2} \mathrm{~d}+\frac{1}{2} \omega+s+\sigma\right)
\end{aligned}
$$

the equation of motion is obtained as

$$
\begin{aligned}
& d * j_{N}=0, j_{N}=p-\mathcal{K} *\left(p+\frac{3}{2} \mathrm{~d}+\frac{1}{2} \omega+s+\sigma\right) \\
& d * j_{N}^{\prime}=0, j_{N}^{\prime}=p^{\prime}+\mathcal{K} *\left(p^{\prime}+\frac{3}{2} \mathrm{~d}^{\prime}+\frac{1}{2} \omega^{\prime}+s^{\prime}+\sigma^{\prime}\right)
\end{aligned}
$$

Similar structure was obtained for a superstring in the AdS space in [42-45]. Since two sets of algebras are decoupled, equations of motion for double coordinates are also decoupled as;

$$
\begin{aligned}
& \mathcal{K}=-1 \Rightarrow \\
& \left\{\begin{array}{l}
\partial_{+}\left(p_{-}+\frac{1}{2}\left(s_{-}+\sigma_{-}+\frac{3}{2} \mathrm{~d}_{-}+\frac{1}{2} \omega_{-}\right)\right)-\frac{1}{2} \partial_{-}\left(s_{+}+\sigma_{+}+\frac{3}{2} \mathrm{~d}_{+}+\frac{1}{2} \omega_{+}\right)=0 \\
\partial_{-}\left(p_{+}^{\prime}+\frac{1}{2}\left(s_{+}^{\prime}+\sigma_{+}^{\prime}+\frac{3}{2} \mathrm{~d}_{+}^{\prime}+\frac{1}{2} \omega_{+}^{\prime}\right)\right)-\frac{1}{2} \partial_{+}\left(s_{-}^{\prime}+\sigma_{-}^{\prime}+\frac{3}{2} \mathrm{~d}_{-}^{\prime}+\frac{1}{2} \omega_{-}^{\prime}\right)=0
\end{array}\right. \\
& \text { or } \\
& \mathcal{K}=1 \Rightarrow \\
& \left\{\begin{array}{l}
\partial_{-}\left(p_{+}+\frac{1}{2}\left(s_{+}+\sigma_{+}+\frac{3}{2} \mathrm{~d}_{+}+\frac{1}{2} \omega_{+}\right)\right)-\frac{1}{2} \partial_{+}\left(s_{-}+\sigma_{-}+\frac{3}{2} \mathrm{~d}_{-}+\frac{1}{2} \omega_{-}\right)=0 \\
\partial_{+}\left(p_{-}^{\prime}+\frac{1}{2}\left(s_{-}^{\prime}+\sigma_{-}^{\prime}+\frac{3}{2} \mathrm{~d}_{-}^{\prime}+\frac{1}{2} \omega_{-}^{\prime}\right)\right)-\frac{1}{2} \partial_{-}\left(s_{+}^{\prime}+\sigma_{+}^{\prime}+\frac{3}{2} \mathrm{~d}_{+}^{\prime}+\frac{1}{2} \omega_{+}^{\prime}\right)=0
\end{array}\right.
\end{aligned}
$$

For $\mathcal{K}=1$ the same dimensional reduction conditions as the previous subsection, $S=S^{\prime}=0$ and $\sigma=\sigma^{\prime}=0$, are imposed. It is possible to choose chiral gauge $\mathrm{d}_{-}=\omega_{-}=0$ and $\mathrm{d}_{+}^{\prime}=\omega_{+}^{\prime}=0$ as shown in [46,47]. Equations of motion are chiral in this gauge, $\partial_{-} j_{N}=0$ and $\partial_{+} j_{N}^{\prime}=0$.

The equations of motion in flat space are the followings. The following lightcone gauge is imposed,

$$
x^{+}=x_{0}^{+}+p^{+}(\sigma+\tau), x^{\prime+}=x_{0}^{\prime+}+p^{+}(\sigma-\tau), \gamma^{+} \theta=\gamma^{+} \theta^{\prime}=0,
$$


with constant $p^{+}$. Then the left/right conserved currents in (5.24) become chiral currents,

$$
\begin{gathered}
\partial_{+} \operatorname{tr}\left(j_{N} P_{\perp m}\right)=0 \Rightarrow \partial_{+}\left(\partial_{-} x^{\perp m}\right)=0, \quad \partial_{+} \operatorname{tr}\left(j_{N} D_{\mu}\right)=0 \Rightarrow \partial_{+}\left(p^{+} \gamma^{-} \theta\right)_{\mu}=0 \\
\partial_{-} \operatorname{tr}\left(j_{N}^{\prime} P_{\perp m^{\prime}}\right)=0 \Rightarrow \partial_{-}\left(\partial_{-} x^{\perp m^{\prime}}\right)=0, \quad \partial_{-} \operatorname{tr}\left(j_{N}^{\prime} D_{\mu^{\prime}}\right)=0 \Rightarrow \partial_{-}\left(p^{+} \gamma^{-} \theta^{\prime}\right)_{\mu^{\prime}}=0
\end{gathered}
$$

The dimensional reduction condition reduces $x$ and $x^{\prime}$ into the usual one, for example $X=x+x^{\prime}$, then equations of motion reduce into the usual one

$$
\partial_{+} \partial_{-} X^{\perp m}=0, \partial_{+} \theta^{\perp \mu}=0, \partial_{-} \theta^{\perp \mu^{\prime}}=0
$$

for the lightcone variables $X^{\perp m}, \theta^{\perp \mu}, \theta^{\perp \mu^{\prime}}$.

\subsection{Type II superstring with R-R extension}

In this subsection we include the R-R central charges $\Upsilon$ and F. For the nondegenerate type II super-Poincaré algebra with R-R central charge we use following notation of currents

$$
\begin{aligned}
& J_{[0]}=\left\{S, S^{\prime}\right\}, \quad J_{\left[\frac{1}{2}\right]}=\left\{D, D^{\prime}\right\}, \quad J_{[1]}=\left\{P, P^{\prime}, \Upsilon, F\right\}, \quad J_{\left[\frac{3}{2}\right]}=\left\{\Omega, \Omega^{\prime}\right\}, \quad J_{[2]}=\left\{\Sigma, \Sigma^{\prime}\right\} \\
& j_{[0]}=\left\{s, s^{\prime}\right\}, \quad j_{\left[\frac{1}{2}\right]}=\left\{\mathrm{d}, \mathrm{d}^{\prime}\right\}, \quad j_{[1]}=\left\{p, p^{\prime}, u, f\right\}, \quad j_{\left[\frac{3}{2}\right]}=\left\{\omega, \omega^{\prime}\right\}, \quad j_{[2]}=\left\{\sigma, \sigma^{\prime}\right\} .
\end{aligned}
$$

The left-invariant currents and the MC equations are

$$
\begin{aligned}
g^{-1} d g & =J+J^{\prime}+\Upsilon+\mathrm{F} \\
J & =S+D+P+\Omega+\Sigma, J^{\prime}=S^{\prime}+D^{\prime}+P^{\prime}+\Omega^{\prime}+\Sigma^{\prime} \\
d S & =-S \wedge S \\
d D & =-S \wedge D-D \wedge S \\
d P & =-S \wedge P-P \wedge S-D \wedge D \\
d \Omega & =-S \wedge \Omega-\Omega \wedge S-D \wedge P-P \wedge D-D^{\prime} \wedge \mathrm{F}-\mathrm{F} \wedge D^{\prime} \\
d \Sigma & =-S \wedge \Sigma-\Sigma \wedge S-D \wedge \Omega-\Omega \wedge D-P \wedge P-\frac{1}{2} \mathrm{~F} \wedge \Upsilon-\frac{1}{2} \Upsilon \wedge \mathrm{F} \\
d \Upsilon & =-\left(S+S^{\prime}\right) \wedge \Upsilon-\Upsilon \wedge\left(S+S^{\prime}\right)-D \wedge D^{\prime}-D^{\prime} \wedge D \\
d \mathrm{~F} & =-\left(S+S^{\prime}\right) \wedge \mathrm{F}-\mathrm{F} \wedge\left(S+S^{\prime}\right) \\
0 & =\Sigma \wedge \Sigma=\Sigma \wedge \Omega+\Omega \wedge \Sigma=\Sigma \wedge P+P \wedge \Sigma=\Sigma \wedge \Upsilon+\Upsilon \wedge \Sigma=\Sigma \wedge \mathrm{F}+\mathrm{F} \wedge \Sigma \\
& =\Sigma \wedge D+D \wedge \Sigma=\Omega \wedge \Omega=\Omega \wedge P+P \wedge \Omega=\Omega \wedge \Upsilon+\Upsilon \wedge \Omega=\Omega \wedge \mathrm{F}+\mathrm{F} \wedge \Omega .
\end{aligned}
$$

The MC equations for primed left-invariant currents are same as the above with replacing unprimed indices with primed indices. Decomposed right-invariant currents and the MC equations are

$$
\begin{aligned}
(d g) g^{-1} & =j+j^{\prime}+u+f \\
j & =s+\mathrm{d}+p+\omega+\sigma, j^{\prime}=s^{\prime}+\mathrm{d}^{\prime}+p^{\prime}+\omega^{\prime}+\sigma^{\prime} \\
d s & =j \wedge j-p \wedge p-\mathrm{d} \wedge \mathrm{d}-\mathrm{d} \wedge(p+\omega)-(p+\omega) \wedge \mathrm{d}+s \wedge(u+f)+(u+f) \wedge s \\
d \mathrm{~d} & =2 \mathrm{~d} \wedge \mathrm{d}+\mathrm{d} \wedge\left(p+\omega+\mathrm{d}^{\prime}+f\right)+\left(p+\omega+\mathrm{d}^{\prime}+f\right) \wedge \mathrm{d} \\
d p & =-\mathrm{d} \wedge \mathrm{d}+2 p \wedge p+\mathrm{d} \wedge p+p \wedge \mathrm{d}
\end{aligned}
$$




$$
\begin{aligned}
d \omega & =\mathrm{d} \wedge(-p+\omega)+(-p+\omega) \wedge \mathrm{d}-\mathrm{d}^{\prime} \wedge f-f \wedge \mathrm{d}^{\prime} \\
d \sigma & =-p \wedge p-\mathrm{d} \wedge \omega-\omega \wedge \mathrm{d}-\frac{1}{2} u \wedge f-\frac{1}{2} f \wedge u \\
d u & =-\mathrm{d} \wedge \mathrm{d}^{\prime}-\mathrm{d}^{\prime} \wedge \mathrm{d}+u \wedge f+f \wedge u \\
d f & =\left(\mathrm{d}+\mathrm{d}^{\prime}\right) \wedge f+f \wedge\left(\mathrm{d}+\mathrm{d}^{\prime}\right)+u \wedge f+f \wedge u \\
0 & =\sigma \wedge \sigma=\sigma \wedge \omega+\omega \wedge \sigma=\sigma \wedge p+p \wedge \sigma=\sigma \wedge u+u \wedge \sigma=\sigma \wedge f+f \wedge \sigma \\
& =\sigma \wedge \mathrm{d}+\mathrm{d} \wedge \sigma=\omega \wedge \omega=\omega \wedge p+p \wedge \omega=\omega \wedge u+u \wedge \omega=\omega \wedge f+f \wedge \omega .
\end{aligned}
$$

The MC equations for primed decomposed right-invariant currents are same as the above with replacing unprimed indices with primed indices.

An action for a superstring in manifestly T-duality formalism is given by

$$
\begin{aligned}
\mathcal{L}_{0} & =-\frac{1}{2} \hat{\operatorname{tr}}\left[P \wedge * P+P^{\prime} \wedge * P^{\prime}\right]=-\frac{1}{2} \operatorname{Tr}\left[P \wedge * P+P^{\prime} \wedge * P^{\prime}\right] \\
\mathcal{L}_{\mathrm{WZ}} & =-\mathcal{K} \operatorname{tr}_{B}\left[\Sigma \wedge S+\Omega \wedge D+\Sigma^{\prime} \wedge S^{\prime}+\Omega^{\prime} \wedge D^{\prime}+\mathrm{F} \wedge \Upsilon\right] \\
& =-\mathcal{K} \operatorname{Tr}\left[\Sigma \wedge S+\Omega \wedge D-\Sigma^{\prime} \wedge S^{\prime}-\Omega^{\prime} \wedge D^{\prime}+\mathrm{F} \wedge \Upsilon\right] .
\end{aligned}
$$

From the MC equations in (5.29)

$$
\begin{aligned}
& \operatorname{tr} \Delta d\left(\sigma-s+\omega-\mathrm{d}+\sigma^{\prime}-s^{\prime}+\omega^{\prime}-\mathrm{d}^{\prime}+f-u\right) \\
& \quad=-\operatorname{tr} \Delta d\left(j+j^{\prime}+\frac{1}{2}\left(-\omega+\mathrm{d}-\omega^{\prime}+\mathrm{d}^{\prime}\right)+2 u\right) \\
& \quad=-\operatorname{tr} \Delta d\left(p+\frac{3}{2} \mathrm{~d}+\frac{1}{2} \omega+s+\sigma+p^{\prime}+\frac{3}{2} \mathrm{~d}^{\prime}+\frac{1}{2} \omega^{\prime}+s^{\prime}+\sigma^{\prime}+2 u\right)
\end{aligned}
$$

the equation of motion is obtained as

$$
\operatorname{tr} \Delta d *\left(p+p^{\prime}-\mathcal{K} *\left(p+\frac{3}{2} \mathrm{~d}+\frac{1}{2} \omega+s+\sigma+p^{\prime}+\frac{3}{2} \mathrm{~d}^{\prime}+\frac{1}{2} \omega^{\prime}+s^{\prime}+\sigma^{\prime}+2 u\right)\right)=0
$$

After imposing the constraint $\Upsilon=0$ leading to $u=0$ in addition to $S=S^{\prime}=0$ and $\sigma=\sigma^{\prime}=0$ constraints, equations of motion reduces to the one obtained in (5.25).

\section{Conclusions and discussions}

We present a superspace formulation of type II superstring background with manifest T-duality symmetry including $\mathrm{R}-\mathrm{R}$ gauge fields. The nondegenerate super-Poincaré affine algebra with the central extension is given in (2.6) and (2.7) to define the superspace. The $\kappa$-symmetric Virasoro constraints are obtained. It determines torsion constraints confirming the consistency with the Bianchi identities. Obtained torsion constraints are much simpler than the previous case [18]: all torsions with dimension 1 and less are trivial. The vielbein superfield $E_{\underline{A M}}$ is identified with the supergravity multiplet. The $\mathrm{R}-\mathrm{R}$ gauge fields are $C_{\mathrm{RR} \underline{a}} \alpha \alpha^{\prime}=E_{\underline{a} \mathrm{~F}}$ and R-R gauge field strengths are $F_{\mathrm{RR}} \alpha^{\prime} \underline{b c}=E_{\mathrm{F} \Sigma}$ as well as $F_{\mathrm{RR}}{ }^{\alpha \beta^{\prime}}=E_{\Omega \Omega^{\prime}}$, while NS-NS gauge field strengths are $F_{\mathrm{NS} \underline{a}} \underline{b c}=E_{\underline{a} \Sigma}$ as well as $F_{\mathrm{NS}}{ }^{\alpha \beta}=E_{\Omega \Omega}$ and $F_{\mathrm{NS}}{ }^{\alpha^{\prime} \beta^{\prime}}=E_{\Omega^{\prime} \Omega^{\prime}}$. 
A Green-Schwarz superstring action is given where the Wess-Zumino term is written by bilinears of left-invariant currents locally. Therefore the Noether current is obtained easily. Left and right moving currents are separated by the auxiliary Lorentz coordinates. Auxiliary degrees of freedom of the space are removed by dimensional reduction conditions in addition to the section condition.

It is also possible to write down an action in the Hamiltonian formalism for a superstring in manifestly T-duality formalism with manifest $\kappa$ symmetry is given as

$$
\begin{aligned}
\mathcal{L}= & \partial_{\tau} Z^{\underline{M}} \partial_{\underline{M}}-\chi_{\tau} \mathcal{H}_{\tau}-\chi_{\sigma} \mathcal{H}_{\sigma}-\chi_{\alpha} \mathcal{B}^{\alpha}-\chi_{\alpha^{\prime}} \mathcal{B}^{\alpha^{\prime}} \\
& -\chi_{1} \mathcal{C}-\chi_{2} \mathcal{D}-\chi_{3} \mathcal{C}^{\prime}-\chi_{4} \mathcal{D}+\chi_{S} S+\chi_{S^{\prime}} S^{\prime}+\chi_{\Upsilon} \Upsilon
\end{aligned}
$$

$\chi$ 's are all multipliers of the constraints. The section condition and the strong condition in the doubled coordinate space are

$$
\partial^{\underline{M}} \partial_{\underline{M}} \Psi\left(Z^{\underline{L}}\right)=\partial^{\underline{M}} \Phi\left(Z^{\underline{L}}\right) \partial_{\underline{M}} \Psi\left(Z^{\underline{L}}\right)=0
$$

for physical states $\Phi\left(Z^{\underline{L}}\right)$ and $\Psi\left(Z^{\underline{L}}\right)$.

There are many interesting questions which should be clarified such as structure of supersymmetric AdS space, branes and exotic branes in the superspace and the appearance of the minimum length.

\section{Acknowledgments}

W.S. thanks to Martin Poláček and M.H. thanks to Jeong-Hyuck Park for valuable discussions. M.H. and K.K. also thanks Yukawa Institute for Theoretical Physics at Kyoto University for useful talks and discussions at the YITP workshop on "Exotic Structures of Spacetime" in March, 2014. M.H. would like to thank the Simons Center for Geometry and Physics for hospitality during "the 2014 Summer Simons workshop in Mathematics and Physics" where this work has been developed. The work of M.H. is supported by Grant-in-Aid for Scientific Research (C) No. 24540284 from The Ministry of Education, Culture, Sports, Science and Technology of Japan, and the work of W.S. is supported in part by National Science Foundation Grant No. PHY-1316617.

Open Access. This article is distributed under the terms of the Creative Commons Attribution License (CC-BY 4.0), which permits any use, distribution and reproduction in any medium, provided the original author(s) and source are credited.

\section{References}

[1] W. Siegel, Two vierbein formalism for string inspired axionic gravity, Phys. Rev. D 47 (1993) 5453 [hep-th/9302036] [InSPIRE].

[2] W. Siegel, Superspace duality in low-energy superstrings, Phys. Rev. D 48 (1993) 2826 [hep-th/9305073] [INSPIRE].

[3] W. Siegel, Manifest duality in low-energy superstrings, hep-th/9308133 [INSPIRE]. 
[4] N. Hitchin, Generalized Calabi-Yau manifolds, Quart. J. Math. Oxford Ser. 54 (2003) 281 [math/0209099] [INSPIRE].

[5] M. Gualtieri, Generalized complex geometry, math/0401221 [INSPIRE].

[6] S.F. Hassan, $\mathrm{SO}(d, d)$ transformations of Ramond-Ramond fields and space-time spinors, Nucl. Phys. B 583 (2000) 431 [hep-th/9912236] [INSPIRE].

[7] M. Fukuma, T. Oota and H. Tanaka, Comments on T dualities of Ramond-Ramond potentials on tori, Prog. Theor. Phys. 103 (2000) 425 [hep-th/9907132] [INSPIRE].

[8] O. Hohm, S.K. Kwak and B. Zwiebach, Unification of type II strings and T-duality, Phys. Rev. Lett. 107 (2011) 171603 [arXiv:1106.5452] [INSPIRE].

[9] I. Jeon, K. Lee and J.-H. Park, Ramond-Ramond cohomology and $O(D, D)$ T-duality, JHEP 09 (2012) 079 [arXiv:1206.3478] [INSPIRE].

[10] I. Jeon, K. Lee, J.-H. Park and Y. Suh, Stringy unification of type IIA and IIB supergravities under $N=2 D=10$ supersymmetric double field theory, Phys. Lett. B 723 (2013) 245 [arXiv: 1210.5078] [INSPIRE].

[11] C.M. Hull, A geometry for non-geometric string backgrounds, JHEP 10 (2005) 065 [hep-th/0406102] [INSPIRE].

[12] C.M. Hull, Doubled geometry and T-folds, JHEP 07 (2007) 080 [hep-th/0605149] [INSPIRE].

[13] C.M. Hull, Generalised geometry for M-theory, JHEP 07 (2007) 079 [hep-th/0701203] [INSPIRE].

[14] M. Graña, Flux compactifications in string theory: a comprehensive review, Phys. Rept. 423 (2006) 91 [hep-th/0509003] [INSPIRE].

[15] G. Aldazabal, D. Marques and C. Núñez, Double field theory: a pedagogical review, Class. Quant. Grav. 30 (2013) 163001 [arXiv: 1305.1907] [INSPIRE].

[16] O. Hohm, D. Lüst and B. Zwiebach, The spacetime of double field theory: review, remarks and outlook, Fortsch. Phys. 61 (2013) 926 [arXiv:1309.2977] [INSPIRE].

[17] D.S. Berman, M. Cederwall and M.J. Perry, Global aspects of double geometry, JHEP 09 (2014) 066 [arXiv:1401.1311] [INSPIRE].

[18] M. Hatsuda, K. Kamimura and W. Siegel, Superspace with manifest T-duality from type-II superstring, JHEP 06 (2014) 039 [arXiv:1403.3887] [INSPIRE].

[19] M. Poláček and W. Siegel, T-duality off shell in 3D type II superspace, JHEP 06 (2014) 107 [arXiv: 1403.6904] [INSPIRE].

[20] P.S. Howe, Supergravity in superspace, Nucl. Phys. B 199 (1982) 309 [inSPIRE].

[21] P.S. Howe and P.C. West, The complete $N=2, D=10$ supergravity, Nucl. Phys. B 238 (1984) 181 [inSPIRE].

[22] N. Berkovits and P.S. Howe, Ten-dimensional supergravity constraints from the pure spinor formalism for the superstring, Nucl. Phys. B 635 (2002) 75 [hep-th/0112160] [INSPIRE].

[23] W. Siegel, Covariant approach to superstrings, in Symposium on anomalies, geometry, topology, Chicago March 27-30 1985, W.A. Bardeen and A.R. White eds., World Scientific, Singapore (1985), pg. 348 [INSPIRE].

[24] W. Siegel, Covariant superstrings, in Unified string theories, Santa Barbara July 29-August 16 1985, M. Green and D. Gross eds., World Scientific, Singapore (1985), pg. 559 [INSPIRE]. 
[25] W. Siegel, Classical superstring mechanics, Nucl. Phys. B 263 (1986) 93 [inSPIRE].

[26] W. Siegel, Randomizing the superstring, Phys. Rev. D 50 (1994) 2799 [hep-th/9403144] [INSPIRE].

[27] M. Hatsuda and K. Kamimura, Classical AdS superstring mechanics, Nucl. Phys. B 611 (2001) 77 [hep-th/0106202] [INSPIRE].

[28] M. Hatsuda, Sugawara form for AdS superstring, Nucl. Phys. B 730 (2005) 364 [hep-th/0507047] [INSPIRE].

[29] S. Bonanos, J. Gomis, K. Kamimura and J. Lukierski, Maxwell superalgebra and superparticle in constant gauge backgrounds, Phys. Rev. Lett. 104 (2010) 090401 [arXiv:0911.5072] [INSPIRE].

[30] W. Siegel, New superspaces/algebras for superparticles/strings, arXiv:1106.1585 [INSPIRE].

[31] M. Poláček and W. Siegel, Natural curvature for manifest T-duality, JHEP 01 (2014) 026 [arXiv: 1308.6350] [INSPIRE].

[32] P.K. Townsend, Supersymmetric extended solitons, Phys. Lett. B 202 (1988) 53 [inSPIRE].

[33] P.K. Townsend, P-brane democracy, in The world in eleven dimensions, M.J. Duff ed., (1999), pg. 375 [hep-th/9507048] [INSPIRE].

[34] J.A. de Azcarraga, J.P. Gauntlett, J.M. Izquierdo and P.K. Townsend, Topological extensions of the supersymmetry algebra for extended objects, Phys. Rev. Lett. 63 (1989) 2443 [InSPIRE].

[35] W. Siegel, Free field equations for everything, Conf. Proc. C 870311 (1987) 13 [InSPIRE].

[36] M. Hatsuda and T. Kimura, Canonical approach to Courant brackets for D-branes, JHEP 06 (2012) 034 [arXiv:1203.5499] [INSPIRE].

[37] M. Hatsuda and K. Kamimura, SL(5) duality from canonical M2-brane, JHEP 11 (2012) 001 [arXiv: 1208.1232] [INSPIRE].

[38] M. Hatsuda and K. Kamimura, M5 algebra and SO(5,5) duality, JHEP 06 (2013) 095 [arXiv: 1305.2258] [INSPIRE].

[39] E. Witten, Twistor-like transform in ten-dimensions, Nucl. Phys. B 266 (1986) 245 [INSPIRE].

[40] J.A. Shapiro and C.C. Taylor, Supergravity torsion constraints from the $10 D$ superparticle, Phys. Lett. B 181 (1986) 67 [InSPIRE].

[41] J.A. Shapiro and C.C. Taylor, Superspace supergravity from the superstring, Phys. Lett. B 186 (1987) 69 [INSPIRE].

[42] M. Hatsuda and K. Yoshida, Classical integrability and super Yangian of superstring on $A d S_{5} \times S^{5}, A d v$. Theor. Math. Phys. 9 (2005) 703 [hep-th/0407044] [INSPIRE].

[43] M. Hatsuda and K. Yoshida, Super Yangian of superstring on $A d S_{5} \times S^{5}$ revisited, Adv. Theor. Math. Phys. 15 (2011) 1485 [arXiv:1107.4673] [INSPIRE].

[44] M. Hatsuda, Sugawara form for AdS superstring, Nucl. Phys. B 730 (2005) 364 [hep-th/0507047] [INSPIRE].

[45] I. Bena, J. Polchinski and R. Roiban, Hidden symmetries of the $A d S_{5} \times S^{5}$ superstring, Phys. Rev. D 69 (2004) 046002 [hep-th/0305116] [INSPIRE]. 
[46] S.J. Gates Jr. et al., Lorentz covariant quantization of the heterotic superstring, Phys. Lett. B 225 (1989) 44 [INSPIRE].

[47] M. Hatsuda and Y. Michishita, Kappa symmetric OSp(2|2) WZNW model, JHEP 06 (2008) 049 [arXiv:0804.1831] [INSPIRE].

[48] M. Hatsuda, K Kamimura and W Siegel, work in progress. 